TRANSACTIONS OF THE

AMERICAN MATHEMATICAL SOCIETY

Volume 235, January 1978

\title{
SPECTRAL PROPERTIES OF TENSOR PRODUCTS OF LINEAR OPERATORS. I
}

\author{
BY
}

TAKASHI ICHINOSE

\begin{abstract}
The aim of the present paper is to obtain, for tensor products of linear operators, their essential spectra in the sense of F. E. Browder, F. Wolf and $M$. Schechter and explicit formulae of their nullity, deficiency and index. The theory applies to $A \otimes I+I \otimes B$ and $A \otimes B$.
\end{abstract}

Introduction. Let $A$ and $B$ be densely defined closed linear operators in complex Banach spaces $X$ and $Y$ respectively with domains $D[A]$ and $D[B]$ and with nonempty resolvent sets $\rho(A)$ and $\rho(B)$. Associated with each polynomial of degrees $m$ in $\xi$ and $n$ in $\eta$

$$
P(\xi, \eta)=\sum_{j k} c_{j k} \xi^{j} \eta^{k}
$$

is a polynomial operator

$$
P\{A \otimes I, I \otimes B\} \equiv \sum_{j k} c_{j k} A^{j} \otimes B^{k}
$$

in the tensor product $X \hat{\otimes}_{\alpha} Y$, the completion of $X \otimes Y$ with respect to a quasi-uniform reasonable norm $\alpha$. In particular, the operators $A \otimes I+I \otimes$ $B$ and $A \otimes B$ correspond respectively to the polynomials $\xi+\eta$ and $\xi \eta$. The symbol $I$ stands for the identity operators in both $X$ and $Y$. The domain of (0.2), which is by definition $\bigcap_{j k ; c_{k} \neq 0} D\left[A^{j}\right] \otimes D\left[B^{k}\right]$, can be shown to coincide with $D\left[A^{m}\right] \otimes D\left[B^{n}\right]$. Assume (0.2) is closable in $X \hat{\otimes}_{\alpha} Y$ and denote its closure by $\tilde{P}\{A \otimes I, I \otimes B\}$.

The main concern of the present paper is with the problem of what spectral contributions $A$ and $B$ make to $\tilde{P}\{A \otimes I, I \otimes B\}$. For a certain class of polynomials $P(\xi, \eta)$, the spectrum of $\tilde{P}\{A \otimes I, I \otimes B\}$ has been determined in Ichinose [11] and [12] (cf. Reed and Simon [20]):

$$
\sigma(\tilde{P}\{A \otimes I, I \otimes B\})=P(\sigma(A), \sigma(B)),
$$

Received by the editors February 27, 1976.

AMS (MOS) subject classifications (1970). Primary 47A60; Secondary 47A10, 47B30.

Key words and phrases. Topological tensor products, operators on tensor products of Banach spaces, separation of variables, spectral mapping theorem, spectrum, essential spectrum, Fredholm operator, nullity, deficiency and index of operators.

Copyright $\odot$ 1978, American Mathematical Society 
where the spectrum is denoted by $\sigma$. The present paper gives exact representions of the essential spectra of $\tilde{P}\{A \otimes I, I \otimes B\}$ in terms of the parts of the spectra of $A$ and $B$. By the essential spectra are meant those in the sense of $\mathrm{F}$. E. Browder [3], F. Wolf [26] and M. Schechter [22]; they are closed subsets in the complex plane $C$ and denoted respectively by $\sigma_{e b}, \sigma_{e w}$ and $\sigma_{e m}$. As by-products, their complementary sets, i.e. the set of all isolated, finite-dimensional eigenvalues and the intersection of the Browder essential spectrum and the Fredholm domain, are also represented. Further, formulae expressing the nullity, deficiency and index for $\tilde{P}\{A \otimes I, I \otimes B\}$ in terms of the quantities concerning $A$ and $B$ are derived. The class of admissible polynomials depends on $A$ and $B$ and therefore on their spectra.

The results may be of use as basic principles in the spectral theory of many-body Schrödinger operators (e.g. Balslev and Combes [1] and B. Simon [23]).

In $\$ 1$ there are given some preliminary results on linear operators and tensor products which play underlying roles in the next sections.

$\$ 2$ is devoted to the study of some useful properties of the admissible polynomials, the class of which is denoted by $\mathscr{P}_{e}(A, B)$ and defined as follows.

A polynomial $P(\xi, \eta)$ is said to belong to $\mathscr{P}_{e}(A, B)$ if it satisfies that $P(\sigma(A), \sigma(B)) \neq \mathrm{C}$ when both $\sigma(A)$ and $\sigma(B)$ are nonempty and that for every $\kappa \notin P(\sigma(A), \sigma(B))$ with $\operatorname{dist}(\kappa, P(\sigma(A), \sigma(B)))>0$ (for every $\kappa \in \mathbf{C}$ when either $\sigma(A)$ or $\sigma(B)$ is empty) there exist nonempty open sets $U$ and $V$ with $C U \subset \rho(A)$ and $C V \subset \rho(B)$ having the following properties:

(i) for each sufficiently large $r>0$, the restrictions of the boundaries $\partial U$ and $\partial V$ to the closed disc $K_{r}=\{\zeta ;|\zeta|<r\}$ consist of a finite number of rectifiable Jordan arcs and have a length $O(r)$ as $r \rightarrow \infty$;

(ii) $\operatorname{dist}(\kappa, P(U, V))>0$;

(iii) $\left\|\xi(\xi I-A)^{-1}\right\|$ is uniformly bounded on $C U$ and $\left\|\eta(\eta I-B)^{-1}\right\|$ is uniformly bounded on $\mathrm{C} V$;

(iv) for some $\tau>0,|P(\xi, \eta)|(|\xi|+|\eta|)^{-\tau}$ is bounded away from zero on $\bar{U} \times \bar{V}$ for sufficiently large $|\xi|+|\eta|$.

Note that if both $A$ and $B$ are bounded every polynomial belongs to $\mathscr{P}_{e}(A, B) . \S 3$ contains the main results. A full use of the properties of the polynomials in $\mathscr{P}_{e}(A, B)$ studied in $\$ 2$ proves the theorems on the Browder and Wolf essential spectra of $\tilde{P}\{A \otimes I, I \otimes B\}$ that for $P \in \mathcal{P}_{e}(A, B)$

$$
\begin{aligned}
& \sigma_{e b}(\tilde{P}\{A \otimes I, I \otimes B\})=P\left(\sigma_{e b}(A), \sigma(B)\right) \cup P\left(\sigma(A), \sigma_{e b}(B)\right) ; \\
& \sigma_{e w}(\tilde{P}\{A \otimes I, I \otimes B\})=P\left(\sigma_{e w}(A), \sigma(B)\right) \cup P\left(\sigma(A), \sigma_{e w}(B)\right) .
\end{aligned}
$$

On the proof of the inclusion $C$ it works that $P(\xi, \eta)$ belongs to $\mathscr{P}_{e}(A, B)$ which implies that $|P(\xi, \eta)|$ becomes large on $\sigma(A) \times \sigma(B)$ with $|\xi|+|\eta|$ so 
that it prevents "cancellations" at infinity. The other inclusion $\supset$, however, is valid in fact for every polynomial $P(\xi, \eta)$. A further elaboration enables one to derive formulae of the nullity, deficiency and index for $\tilde{P}\{A \otimes I, I \otimes B\}$. With this very index the Schechter essential spectrum of $P\{A \otimes I, I \otimes B\}$ is also exactly determined. It will be also observed that, if, in addition, the crossnorm $\alpha$ is faithful, then all the results are valid for another associated polynomial operator

$$
P[A \otimes I, I \otimes B] \equiv \sum_{j k} c_{j k} A^{j} \hat{\otimes}_{\alpha} B^{k}
$$

in $X \hat{\otimes}_{\alpha} Y$, where the $A^{j} \hat{\otimes}_{\alpha} B^{k}$ are the closures of the $A^{j} \otimes B^{k}$ since they are closable (see [12]).

$\$ 4$ gives a concise summary of the main results, in particular, in two special cases for the polynomials $\xi+\eta$ and $\xi \eta$, which may be of importance in applications. The polynomial $\xi+\eta$ belongs to $\mathscr{P}_{e}(A, B)$ if the spectra of $A$ and $B$ are included respectively in the sectors

$$
S\left(\theta_{A}\right)=\left\{\xi \in \mathbf{C} ;|\arg \xi| \leqslant \theta_{A}\right\} \text { and } S\left(\theta_{B}\right)=\left\{\eta \in \mathbf{C} ;|\arg \eta|<\theta_{B}\right\}
$$

with $0 \leqslant \theta_{A}+\theta_{B}<\pi$ and if

$$
\begin{aligned}
\left\|\xi(\xi I-A)^{-1}\right\| \leqslant M_{A}(\arg \xi), & \xi \notin S\left(\theta_{A}\right), \\
\left\|\eta(\eta I-B)^{-1}\right\| \leqslant M_{B}(\arg \eta), & \eta \notin S\left(\theta_{B}\right),
\end{aligned}
$$

where $M_{T}(\theta)$ is a constant depending only on $T$ and $\theta$. The polynomial $\xi \eta$ belongs to $\mathscr{P}_{e}(A, B)$ if it does not occur that one of the extended spectra of $A$ and $B$ contains 0 while the other contains $\infty$.

An exact representation of the essential spectrum of $\tilde{P}\{A \otimes I, I \otimes B\}$ in the sense of T. Kato [15] will be given in a forthcoming paper.

For the basic notions and results on linear operators and tensor products used here see T. Kato [15] and R. Schatten [21] (see also [5], [14], [24], [25] and [7], [8]).

\section{Preliminary results.}

1.1. Essential spectra of linear operators. Let $Z$ be a complex Banach space with $Z$ ' its topological dual space. Let $T$ be a closed linear operator with both domain $D[T]$ and range $R[T]$ in $Z$. The identity operator in $Z$ is denoted by $I$. For $T$ densely defined, the adjoint of $T$ is denoted by $T^{\prime}$. We denote the spectrum and resolvent set of $T$ by $\sigma(T)$ and $\rho(T)$, respectively.

The nullity of $T$, null $T$, is the dimension of the null space $N[T]$ of $T$. The deficiency of $T$, def $T$, is the dimension of $Z / R[T]$. The index of $T$, ind $T$, is defined as ind $T=\operatorname{nul} T-\operatorname{def} T$, if at least one of null $T$ and def $T$ is finite. $T$ is said to be semi-Fredholm if $R[T]$ is closed and at least one of null $T$ and 
def $T$ is finite. In addition, if both nul $T$ and def $T$ are finite, $T$ is said to be Fredholm.

The semi-Fredholm (resp. Fredholm) domain of $T$, denoted by $\rho_{e k}(T)$ (resp. $\rho_{\text {ew }}(T)$ ), is by definition the set of all $\lambda$ in the complex plane $\mathbf{C}$ for which $T-\lambda I$ is semi-Fredholm (resp. Fredholm).

We shall use also the notions of the approximate nullity and approximate deficiency of $T$, which are denoted by $\operatorname{nul}^{\prime} T$ and $\operatorname{def}^{\prime} T$, respectively. It is known that nul' $T$ (resp. def' $T$ ) coincides with nul $T$ (resp. def $T$ ) if $R[T]$ is closed, and is infinite if $R[T]$ is not closed [15, IV, $\S 5$, Theorem 5.10]. nul' $T$ is positive if and only if there is a sequence $\left\{z_{l}\right\}_{l=1}^{\infty} \subset D[T]$ of unit vectors with $T z_{l} \rightarrow 0$ as $l \rightarrow \infty$, and nul' $T$ is infinite if and only if this sequence $\left\{z_{l}\right\}_{l=1}^{\infty}$ of unit vectors can be chosen to be noncompact ([15, IV, §5, Theorem 5.11] and [26]). Here we say a sequence $\left\{z_{l}\right\}_{l=1}^{\infty} \subset Z$ is compact if every subsequence of it contains a convergent subsequence.

The approximate point spectrum of $T, \sigma_{\pi}(T)$, is the set of all $\lambda$ in $\mathbf{C}$ such that $\operatorname{nul}^{\prime}(T-\lambda I)>0$. The point spectrum of $T, \sigma_{p}(T)$, is a subset of $\sigma_{\pi}(T)$. Let $\sigma_{+}(T)$ (resp. $\sigma_{-}(T)$ ) be the set of all $\lambda$ in $\mathbf{C}$ such that $\operatorname{nul}^{\prime}(T-\lambda I)=\infty$ (resp. $\left.\operatorname{def}^{\prime}(T-\lambda I)=\infty\right)$.

T. Kato [15], F. Wolf [26], F. E. Browder [3] and M. Schechter [22] have defined the essential spectrum of $T$ differently.

The Kato essential spectrum of $T, \sigma_{e k}(T)$, is the complementary set in $\mathbf{C}$ of the semi-Fredholm domain $\rho_{e k}(T)$ for $T$. The Wolf essential spectrum of $T$, $\sigma_{e w}(T)$, is the complementary set in $\mathbf{C}$ of the Fredholm domain $\rho_{e w}(T)$ for $T$. It is easily seen that $\sigma_{e k}(T)=\sigma_{+}(T) \cap \sigma_{-}(T)$ and $\sigma_{e w}(T)=\sigma_{+}(T) \cup$ $\sigma_{-}(T)$. The Schechter essential spectrum of $T, \sigma_{e m}(T)$, is the union of $\sigma_{e w}(T)$ and the set of all $\lambda$ in $\sigma(T)$ for which $T-\lambda I$ is Fredholm with ind $(T-\lambda I)$ $\neq 0$. The Browder essential spectrum of $T, \sigma_{e b}(T)$, is the set of all $\lambda$ in $\sigma(T)$ such that at least one of the following conditions holds: (i) $R[T-\lambda I]$ is not closed; (ii) $\lambda$ is a limit point of $\sigma(T)$; (iii) $\cup_{n>0} N\left[(T-\lambda I)^{n}\right]$ is of infinite dimension.

If $\lambda$ is an isolated point of $\sigma(T)$, by the projection associated with $\lambda$ is meant the bounded linear operator $P$ in $Z$ defined by

$$
P=(2 \pi i)^{-1} \int_{C}(\zeta I-T)^{-1} d \zeta,
$$

where $C$ is a circle round $\lambda$ such that $\lambda$ is the only point of $\sigma(T)$ contained in $C$. If $P$ is finite dimensional, i.e. of finite rank, $\lambda$ is an eigenvalue of $T$ with algebraic multiplicity $t(T ; \lambda)=\operatorname{dim} P$, which is coincident with

$$
\operatorname{dim} \bigcup_{n>0} N\left[(T-\lambda I)^{n}\right]
$$

Such an eigenvalue will be referred to, throughout this paper, as "an isolated, 
finite-dimensional eigenvalue". Then the set $\sigma(T) \backslash \sigma_{e b}(T)$ is the set of all isolated, finite-dimensional eigenvalues of $T$.

All the four essential spectra as above are closed subsets of the complex plane $C$; they are empty if the Banach space $Z$ is of finite dimension. They coincide if $T$ is selfadjoint.

Among these various parts of the spectrum and their boundaries there are the following inclusion relations. The boundary of a set $U$ in the complex plane $\mathbf{C}$ is denoted by $\partial U$.

Proposition 1.1. (a)

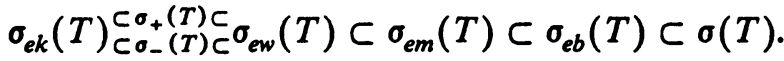

$$
\begin{aligned}
& \sigma_{e k}(T) \cup \sigma_{p}(T)=\sigma_{\pi}(T) \subset \sigma(T) \text {. }
\end{aligned}
$$

$$
\begin{gathered}
\partial \sigma_{e b}(T) \subset \partial \sigma_{e m}(T) \subset \partial \sigma_{e w}(T) \underset{\subset \partial \sigma_{-}(T) \subset \partial \sigma_{e k}(T) .}{\subset \partial \sigma_{+}(T) \subset \sigma} . \\
\partial \sigma(T)=\left(\sigma(T) \backslash \sigma_{e b}(T)\right) \cup \partial \sigma_{e b}(T) \subset \partial \sigma_{z}(T) .
\end{gathered}
$$

Proof. (1.1) and (1.2) are obvious. (1.3) will be shown with the aid of [14, Theorem 6] or [15, IV, $\S 5$, Theorem 5.17]; for $T$ bounded with $D[T]=Z$, it has been shown in D. Miličić and K. Veselić [17]. For (1.4) see e.g. [10]. Q.E.D.

Associated with each polynomial of degree $m$ in $\xi$

$$
p(\xi)=\sum_{j=0}^{m} a_{j} \xi^{j}, \quad a_{m} \neq 0,
$$

is a linear operator

$$
p(T)=\sum_{j=0}^{m} a_{j} T^{j}
$$

in $Z$. If the resolvent set $\rho(T)$ or, more generally, the Fredholm domain $\rho_{\text {ew }}(T)$ of $T$ is not empty, then $p(T)$ is closed with domain $D\left[T^{m}\right]$ (see e.g. [22]). It follows by virtue of the closed graph theorem that the graph norm of $p(T)$ is equivalent to that of $T^{m}$ and hence to the norm

$$
\|z\|+\sum_{j=1}^{m}\left\|T^{j} z\right\|, \quad z \in D\left[T^{m}\right] .
$$

In this case, it will be shown that $p(T)^{\prime}=p\left(T^{\prime}\right)$ it $T$ is densely defined.

The relationships between the various parts of the spectrum of $T$ and those of $p(T)$ are given by the following

Proposition 1.2. Let $T: D[T] \subset Z \rightarrow Z$ be a densely defined closed linear operator with nonempty resolvent set $\rho(T)$. Then 

(a) $\sigma(p(T))=p(\sigma(T))$;
(b) $\sigma_{p}(p(T))=p\left(\sigma_{p}(T)\right)$;
(c) $\sigma_{\pi}(p(T))=p\left(\sigma_{\pi}(T)\right)$;
(d) $\sigma_{+}(p(T))=p\left(\sigma_{+}(T)\right)$;
(e) $\sigma_{-}(p(T))=p\left(\sigma_{-}(T)\right)$;
(f) $\sigma_{e b}(p(T))=p\left(\sigma_{e b}(T)\right)$;
(g) $\sigma_{e w}(p(T))=p\left(\sigma_{e w}(T)\right)$;
(h) $\sigma_{e k}(p(T)) \supset p\left(\sigma_{e k}(T)\right)$;
(i) $\sigma_{e m}(p(T)) \subset p\left(\sigma_{e m}(T)\right)$.

Remark. Proposition 1.2 is valid for $T^{\prime}$ in place of $T$. Further, it will be shown that Proposition 1.2 is true also for $T$ with nonempty Fredholm domain.

Proof of Proposition 1.2 AND Comment. The relations (a), (b) and (c) are well known. The other relations have been shown in B. Gramsch and D. Lay [6], for $T$ bounded with $D[T]=Z$. In (h) and (i), equality does not in general hold; they give also simple examples in which these inclusions are proper. For general $T$, the relation (f) has been shown in R. D. Nussbaum [18] and (g) in E. Balslev and T. W. Gamelin [2]. The relations (g) and (h) follow readily from (d) and (e). The relations (d), (e) and (i) will be shown with the aid of [14, Lemma 341], [5, Theorem 2.1] and the following lemma.

Lemma 1.3. Let $T: D[T] \subset Z \rightarrow Z$ be a (not necessarily densely defined) closed linear operator with nonempty resolvent set $\rho(T)$. Let $m$ be a positive integer. If $\operatorname{nul}^{\prime}(T-\lambda I)>0$ there exists a sequence $\left\{e_{l}\right\}_{l=1}^{\infty} \subset D\left[T^{m}\right]$ of unit vectors such that, for $0 \leqslant j \leqslant m-1, T^{j}(T-\lambda I) e_{l} \rightarrow 0$ as $l \rightarrow \infty$. In addition, if $\operatorname{nul}^{\prime}(T-\lambda I)=\infty$ the sequence $\left\{e_{l}\right\}_{l=1}^{\infty}$ can be chosen to be noncompact.

Proof. Let $\left\{z_{l}\right\}_{l=1}^{\infty} \subset D[T]$ be a sequence of unit vectors with $(T-\lambda I) z_{l}$ $\rightarrow 0$ as $l \rightarrow \infty$. Then setting

$$
e_{l}=\left\|(T-\mu I)^{-(m-1)} z_{l+k}\right\|^{-1}(T-\mu I)^{-(m-1)} z_{l+k}, \quad l=1,2, \ldots,
$$

for some large $k$ fixed and $\mu \in \rho(T)$ fixed will give a desired sequence. Further, if $\operatorname{nul}^{\prime}(T-\lambda I)=\infty$ and if $\left\{z_{l}\right\}_{k=1}^{\infty}$ is noncompact, $\left\{e_{l}\right\}_{l=1}^{\infty}$ is noncompact. Q.E.D.

We shall need the following result, a more precise one than the relation (b) in Proposition 1.2. It is a slight extension of [25, Theorem 5.9-D], and can be shown by modifying the proof as in the proof of [26, Lemma 3.8].

Proposition 1.4. Let $T: D[T] \subset Z \rightarrow Z$ be a linear operator and let $p(\xi)=a \prod_{j=1}^{r}\left(\xi-\mu_{j}\right)^{m_{j}}, a \neq 0$, be a polynomial in $\xi$ with distinct zeros $\mu_{1}, \mu_{2}, \ldots, \mu_{r}$. Then

$$
N[p(T)]=N\left[\left(T_{1}-\mu_{1} I\right)^{m_{1}}\right] \oplus \cdots \oplus N\left[\left(T-\mu_{r} I\right)^{m_{r}}\right]
$$


1.2. Tensor products of Banach spaces. Let $X$ and $Y$ be Banach spaces with $X^{\prime}$ and $Y^{\prime}$ their topological dual spaces.

A norm $\alpha$ on the tensor product $X \otimes Y$ is said to be reasonable if it is a crossnorm on $X \otimes Y$ whose dual norm $\alpha^{\prime}$ is also a crossnorm on $X^{\prime} \otimes Y^{\prime}$. $X \hat{\otimes}_{\alpha} Y$ denotes the completion of $X \otimes Y$ with respect to $\alpha$.

We shall introduce a slightly generalized notion of uniform crossnorms, which is useful in the present work. $L(Z)$ denotes the linear space of all bounded linear operators $T$ of the Banach space $Z$ into itself.

A crossnorm $\alpha$ on $X \otimes Y$ is said to be quasi-uniform with constant $k$ on $X \otimes Y$ if

$$
\alpha((T \otimes S) u) \equiv\|(T \otimes S) u\|_{\alpha} \leqslant k\|T\|\|S\|\|u\|_{\alpha}
$$

for every pair $(T, S) \in L(X) \times L(Y)$ and all $u \in X \otimes Y$. A uniform crossnorm is quasi-uniform with constant $k=1$.

Note that if $\alpha$ is a quasi-uniform reasonable norm on $X \otimes Y$ the dual norm $\alpha^{\prime}$ satisfies

$$
\alpha^{\prime}\left(\left(T^{\prime} \otimes S^{\prime}\right) u^{\prime}\right) \equiv\left\|\left(T^{\prime} \otimes S^{\prime}\right) u^{\prime}\right\|_{\alpha^{\prime}} \leqslant k\left\|T^{\prime}\right\|\left\|S^{\prime}\right\|\left\|u^{\prime}\right\|_{\alpha^{\prime}}
$$

for every pair $(T, S) \in L(X) \times L(Y)$ and all $u^{\prime} \in X^{\prime} \otimes Y^{\prime}$.

The smallest reasonable norm $\varepsilon$ and the greatest one $\pi$ are uniform. The prehilbertian norm $\sigma$ on $X \otimes Y$ with both $X$ and $Y$ Hilbert spaces, which is the norm induced by the inner product $\left(x_{1} \otimes y_{1}, x_{2} \otimes y_{2}\right)=\left(x_{1}, x_{2}\right)\left(y_{1}, y_{2}\right)$, is uniform.

A crossnorm $\alpha(\alpha \geqslant \varepsilon)$ on $X \otimes Y$ is said to be faithful if the natural continuous linear mapping $j_{\varepsilon}^{\alpha}: X \hat{\otimes}_{\alpha} Y \rightarrow X \hat{\otimes}_{\varepsilon} Y$ is one-to-one.

In the following, let $P \in L(X)$ and $Q \in L(Y)$ be continuous projections. Then $P X \otimes Q Y$ is a normed linear subspace of $X \hat{\otimes}_{\alpha} Y$ equipped with the norm $\bar{\alpha}$ induced by the norm $\alpha$ of $X \hat{\otimes}_{\alpha} Y$. We denote the closure of $P X \otimes Q Y$ in $X \hat{\otimes}_{\alpha} Y$ by $P X \hat{\otimes}_{\bar{\alpha}} Q Y$.

We shall also use the notion of $\otimes$-norms [8], which are defined on $X_{0} \otimes Y_{0}$ for each pair of Banach spaces $X_{0}$ and $Y_{0}$. For $\alpha$ a $\otimes$-norm, we denote the norm of $u$ as an element of $X_{0} \otimes Y_{0}$ sometimes by $\alpha\left(u ; X_{0}, Y_{0}\right)$.

Proposition 1.5. (a) If $\alpha$ is a quasi-uniform reasonable norm on $X \otimes Y$ with constant $k$, then $\bar{\alpha}$ is a quasi-uniform reasonable norm on $P X \otimes Q Y$ with constant $k\|P\|\|Q\|$. In this case, $P \hat{\otimes}_{\alpha} Q$ is a continuous projection of $X \hat{\otimes}_{\alpha} Y$ into itself and the range $R\left[P \hat{\otimes}_{\alpha} Q\right]$ of $P \hat{\otimes}_{\alpha} Q$ is the closure of $P X \otimes Q Y$ in $X \hat{\otimes}_{\alpha} Y$ :

$$
R\left[P \hat{\otimes}_{\alpha} Q\right] \equiv\left(P \hat{\otimes}_{\alpha} Q\right)\left(X \hat{\otimes}_{\alpha} Y\right)=P X \hat{\otimes}_{\bar{\alpha}} Q Y .
$$

In addition, if $\alpha$ is faithful on $X \otimes Y$, so is $\bar{\alpha}$ on $P X \otimes Q Y$.

(b) If $\alpha$ is $a \otimes$-norm then $\alpha$ is equivalent to $\bar{\alpha}$ on $P X \otimes Q Y$. 
Proof. (a) First we show $\bar{\alpha}$ is a reasonable norm on $P X \otimes Q Y . \bar{\alpha}$ is induced on $P X \otimes Q Y$ by $\alpha$ and so is a crossnorm on it. To show that the dual norm $\bar{\alpha}^{\prime}$ of $\bar{\alpha}$ is a crossnorm on $(P X)^{\prime} \times(Q Y)^{\prime}$, let $\left(x_{1}^{\prime}, y_{1}^{\prime}\right) \in(P X)^{\prime} \times$ $(Q Y)^{\prime}$. It suffices to show

$$
\bar{\alpha}^{\prime}\left(x_{1}^{\prime} \otimes y_{1}^{\prime}\right) \leqslant\left\|x_{1}^{\prime}\right\|\left\|y_{1}^{\prime}\right\|,
$$

since the reverse inequality is always valid. By the Hahn-Banach theorem there exist $x^{\prime} \in X^{\prime}$ and $y^{\prime} \in Y^{\prime}$ with

$$
\left\|x^{\prime}\right\|=\left\|x_{1}^{\prime}\right\|, \quad\left\langle x_{1}, x^{\prime}\right\rangle=\left\langle x_{1}, x_{1}^{\prime}\right\rangle, \quad x_{1} \in P X,
$$

and

$$
\left\|y^{\prime}\right\|=\left\|y_{1}^{\prime}\right\|, \quad\left\langle y_{1}, y^{\prime}\right\rangle=\left\langle y_{1}, y_{1}^{\prime}\right\rangle, \quad y_{1} \in Q Y .
$$

Then

$$
\begin{aligned}
\bar{\alpha}^{\prime}\left(x_{1}^{\prime} \otimes y_{1}^{\prime}\right) & =\sup \left\{\left|\left\langle u_{1}, x_{1}^{\prime} \otimes y_{1}^{\prime}\right\rangle\right| ; u_{1} \in P X \otimes Q Y, \bar{\alpha}\left(u_{1}\right)<1\right\} \\
& =\sup \left\{\left|\left\langle u_{1}, x^{\prime} \otimes y^{\prime}\right\rangle\right| ; u_{1} \in P X \otimes Q Y, \bar{\alpha}\left(u_{1}\right)<1\right\} \\
& =\sup \left\{\left|\left\langle u, x^{\prime} \otimes y^{\prime}\right\rangle\right| ; u \in X \otimes Y, \alpha(u)<1\right\},
\end{aligned}
$$

which, by definition of the dual norm $\alpha^{\prime}$, equals

$$
\alpha^{\prime}\left(x^{\prime} \otimes y^{\prime}\right)=\left\|x^{\prime}\right\|\left\|y^{\prime}\right\|=\left\|x_{1}^{\prime}\right\|\left\|y_{1}^{\prime}\right\| .
$$

This proves that $\bar{\alpha}$ is reasonable on $P X \otimes Q Y$.

Next, we show that $\bar{\alpha}$ is quasi-uniform on $P X \otimes Q Y$. Let $A_{1} \in L(P X)$ and $B_{1} \in L(Q Y)$, and set $A=A_{1} P$ and $B=B_{1} Q$. Then $A \in L(X)$ and $B \in$ $L(Y)$ with $\|A\|<\left\|A_{1}\right\|\|P\|$ and $\|B\|<\left\|B_{1}\right\|\|Q\|$. If $u_{1} \in P X \otimes Q Y$, so that $\left(A_{1} \otimes B_{1}\right) u_{1} \in P X \otimes Q Y$, we obtain

$$
\begin{aligned}
\bar{\alpha}\left(\left(A_{1} \otimes B_{1}\right) u_{1}\right) & =\bar{\alpha}\left((A \otimes B) u_{1}\right)=\alpha\left((A \otimes B) u_{1}\right) \\
& <k\|A\|\|B\| \alpha\left(u_{1}\right)<k\|P\|\|Q\|\left\|A_{1}\right\|\left\|B_{1}\right\| \bar{\alpha}\left(u_{1}\right),
\end{aligned}
$$

since $\alpha$ is quasi-uniform with constant $k$. Thus $\bar{\alpha}$ is quasi-uniform with constant $k\|P\|\|Q\|$.

Since $\alpha$ is quasi-uniform, $P \hat{\otimes}_{\alpha} Q$ belongs to $L\left(X \hat{\otimes}_{\alpha} Y\right)$ and $\left(P \hat{\otimes}_{\alpha} Q\right)^{2}$ $=P \hat{\otimes}_{\alpha} Q$, that is, $P \hat{\otimes}_{\alpha} Q$ is a continuous projection of $X \hat{\otimes}_{\alpha} Y$ into itself and so $R\left[P \hat{\otimes}_{\alpha} Q\right]$ is a closed subspace of $X \hat{\otimes}_{\alpha} Y$. It is evident that $P X \otimes, Q Y$ is included dense in $R\left[P \hat{\otimes}_{\alpha} Q\right]$, so that the closure of $P X \otimes Q Y$ in $X \hat{\otimes}_{\alpha} Y$ coincides with $R\left[P \otimes_{\alpha} Q\right]$.

The last assertion for $\alpha$ faithful is evident.

(b) First note the induced norm $\bar{\alpha}$ is defined precisely by

$$
\bar{\alpha}(u)=\alpha\left(\left(j_{P} \otimes j_{Q}\right) u\right), \quad u \in P X \otimes Q Y,
$$

where $j_{P}$ (resp. $j_{Q}$ ) is the injection of $P X$ (resp. $Q Y$ ) into $X$ (resp. $Y$ ). We have to show $\bar{\alpha}(u)$ is equivalent to $\alpha(u ; P X, Q Y)$. 
For $u \in P X \otimes Q Y$ we have

$$
\begin{aligned}
& \bar{\alpha}(u)=\alpha\left(\left(j_{P} \otimes j_{Q}\right) u ; X, Y\right)<\left\|j_{P}\right\|\left\|j_{Q}\right\| \alpha(u ; P X, Q Y)=\alpha(u ; P X, Q Y), \\
& \alpha(u ; P X, Q Y)=\alpha\left((P \otimes Q)\left(j_{P} \otimes j_{Q}\right) u ; P X, Q Y\right) \\
&<\|P\|\|Q\| \alpha\left(\left(j_{P} \otimes j_{Q}\right) u ; X, Y\right)=\|P\|\|Q\| \bar{\alpha}(u) . \text { Q.E.D. }
\end{aligned}
$$

Note that in Proposition 1.5, if $P$ or $Q$ is of finite rank then $R\left[P \hat{\otimes}_{\alpha} Q\right]=$ $P X \otimes Q Y$.

2. Polynomial operators. In this section we shall study the properties of the polynomials in the class $\mathscr{P}_{e}(A, B)$ defined in the Introduction, which are useful in the next section.

Throughout this section and the next, $X$ and $Y$ are complex Banach spaces and $\alpha$ is a reasonable norm on $X \otimes Y$ unless otherwise specified. Let $A$ : $D[A] \subset X \rightarrow X$ and $B: D[B] \subset Y \rightarrow Y$ be densely defined closed linear operators with nonempty resolvent sets $\rho(A)$ and $\rho(B)$. The identity operators in both $X$ and $Y$ are denoted by the same $I$.

It is assumed that both $\operatorname{dim} X$ and $\operatorname{dim} Y$ are positive and at least one of them is infinite. Only polynomials (0.1) of degrees $m>1$ in $\xi$ and $n>1$ in $\eta$ are considered. For convenience in treating the essential spectra we assume the operator (0.2) associated with (0.1) is closable in $X \hat{\otimes}_{\alpha} Y$ with closure $\tilde{P}\{A \otimes I, I \otimes B\}$. This is the case, for instance, if $\alpha$ is faithful on $X \otimes Y$ [12, Theorem 1.1]. For general $\alpha$ it is open whether or not (0.2) is closable. In fact, it is possible that the norm $\pi$ is not faithful on $X \otimes Y$ for some pairs of Banach spaces $X$ and $Y$, for there is a Banach space without the approximation property, according to $P$. Enflo [4].

To simplify the notation we of ten write

$$
\mathbf{P}_{\lambda}=\tilde{P}\{A \otimes I, I \otimes B\}-\lambda I \hat{\otimes}_{\alpha} I, \quad \mathbf{P}=\mathbf{P}_{0} .
$$

For a polynomial $P(\xi, \eta)$ and subsets $\sigma_{A}, \sigma_{B}$ of $\sigma(A), \sigma(B)$, respectively, we understand $P\left(\sigma_{A}, \sigma_{B}\right)=\varnothing$ if either $\sigma_{A}$ or $\sigma_{B}$ is empty, while otherwise it offers no problem to define $P\left(\sigma_{A}, \sigma_{B}\right)$. For $r>0, K_{r}$ denotes the closed disc $\{\zeta$; $|\zeta|<r\}$.

It is easy to see that if $P(\xi, \eta)$ is in $\mathscr{P}_{e}(A, B)$ the set $P(\sigma(A), \sigma(B))$ is closed in $\mathrm{C}$. The class $\mathscr{P}(A, B)$ introduced in [12] is included in $\mathcal{P}_{e}(A, B)$.

We shall use the following result on the spectrum of $P\{A \otimes I, I \otimes B\}$. It has been shown in [12] for $P \in \mathscr{P}(A, B)$, but the same proof as there is valid in the present case.

THEOREM 2.1. Let $\alpha$ be a quasi-uniform reasonable norm on $X \otimes Y$ and let $P \in \mathcal{P}_{e}(A, B)$. Then

$$
\sigma(\tilde{P}\{A \otimes I, I \otimes B\})=P(\sigma(A), \sigma(B)) .
$$


By this is meant that (2.2) holds valid if both $\sigma(A)$ and $\sigma(B)$ are nonempty, and further that the spectrum of $\tilde{P}\{A \otimes I, I \otimes B\}$ is empty if and only if either $\sigma(A)$ or $\sigma(B)$ is empty.

Now we observe some properties of the polynomials in $\mathscr{P}_{e}(A, B) . P\left\{A^{\prime} \otimes\right.$ $\left.I^{\prime}, I^{\prime} \otimes B^{\prime}\right\}$ stands for the operator (0.2) with $A^{\prime}, B^{\prime}$ and $I^{\prime}$ in place of $A, B$ and $I$, respectively; it is considered as an operator with domain $D\left[\left(A^{\prime}\right)^{m}\right] \otimes$ $D\left[\left(B^{\prime}\right)^{n}\right]$ in $\left(X \hat{\otimes}_{\alpha} Y\right)^{\prime}$ as well as in $X^{\prime} \hat{\otimes}_{\alpha^{\prime}} Y^{\prime}$.

When either $X$ or $Y$ is of finite dimension, all reasonable norms on $X \otimes Y$ are equivalent, and hence quasi-uniform and faithful. We have $X \hat{\otimes}_{\alpha} Y=$ $X \otimes Y$, in which (0.2) is closable, and $\left(X \hat{\otimes}_{\alpha} Y\right)^{\prime}=X^{\prime} \hat{\otimes}_{\alpha^{\prime}} Y^{\prime}=X^{\prime} \otimes Y^{\prime}$.

Proposition 2.2. Assume either $X$ or $Y$, say $Y$, is of finite dimension. Let $P(\xi, \eta)$ be a polynomial $(0.1)$ in $\mathscr{P}_{e}(A, B)$. Then

(a) for each fixed $\nu \in \sigma(B)$

$$
D[P(A, \nu)]=D\left[A^{m}\right], \quad D\left[P\left(A^{\prime}, \nu\right)\right]=D\left[\left(A^{\prime}\right)^{m}\right]
$$

(b) for every positive integer $t$

$$
\begin{gathered}
D\left[P\{A \otimes I, I \otimes B\}^{t}\right]=D\left[A^{t m}\right] \otimes D\left[B^{t n}\right], \\
D\left[P\left\{A^{\prime} \otimes I^{\prime}, I^{\prime} \otimes B^{\prime}\right\}^{t}\right]=D\left[\left(A^{\prime}\right)^{t m}\right] \otimes D\left[\left(B^{\prime}\right)^{\imath n}\right] ;
\end{gathered}
$$

(c) $\tilde{P}\{A \otimes I, I \otimes B\}=P\{A \otimes I, I \otimes B\}$,

$$
\tilde{P}\{A \otimes I, I \otimes B\}^{\prime}=P\{A \otimes I, I \otimes B\}^{\prime}=P\left\{A^{\prime} \otimes I^{\prime}, I^{\prime} \otimes B^{\prime}\right\} ;
$$

(d) for every $\nu$ and every $\lambda$ there exists a constant $C$ such that for all $u \in D\left[A^{i m}\right] \otimes Y$

$$
\left\|\left[(P(A, v)-\lambda I)^{t} \otimes I\right] u\right\|_{\alpha} \leqslant C\left[\left\|P\{A \otimes I, I \otimes B\}^{t} u\right\|_{\alpha}+\|u\|_{\alpha}\right]
$$

and for all $u^{\prime} \in D\left[\left(A^{\prime}\right)^{\prime m}\right] \otimes Y^{\prime}$

$$
\left\|\left[\left(P\left(A^{\prime}, \nu\right)-\lambda I^{\prime}\right)^{t} \otimes I^{\prime}\right] u^{\prime}\right\|_{\alpha^{\prime}} \leqslant C\left[\left\|P\left\{A^{\prime} \otimes I^{\prime}, I^{\prime} \otimes B^{\prime}\right\}^{t} u^{\prime}\right\|_{\alpha^{\prime}}+\left\|u^{\prime}\right\|_{\alpha^{\prime}}\right] .
$$

Proof. (a) Let $\nu \in \sigma(B)$. It is obvious if $\sum_{k=0}^{n} c_{m k} \nu^{k}$ does not vanish. If it vanishes we have only to show $A$ is bounded; note it implies by closedness $D\left[A^{m}\right]=X$ and $D\left[\left(A^{\prime}\right)^{m}\right]=X^{\prime}$. Let $\lambda \notin P(\sigma(A), \sigma(B))$. Since $P \in$ $\mathscr{P}_{e}(A, B)$ there are nonempty open sets $U \supset \sigma(A)$ and $V \supset \sigma(B)$ such that $\lambda \notin P(U, V)$ and $\xi(\xi I-A)^{-1}$ is uniformly bounded in $C U$. In view of [15, III, §6, Theorem 6.13] it suffices to show $U$ is bounded. To see it choose a small $\delta>0$ with $\{\eta ;|\eta-\nu| \leqslant \delta\} \subset V$ such that $\sum_{k=0}^{n} c_{m k} \eta^{k}$ does not vanish on the circumference $|\eta-\nu|=\delta$. There is a large $r_{0}>0$ such that for each fixed $\xi$ with $|\xi|>r_{0}$ the polynomial in $\eta, \sum_{k=0}^{n} c_{m k} \eta^{k}-\lambda \xi^{-m}$, has a zero in the open disc $|\eta-\nu|<\delta$. If $U$ is unbounded we can choose a large $\xi_{0}$ in $U$ 
such that

$$
\left|\sum_{k=0}^{n} c_{m k} \eta^{k}-\lambda \xi_{0}^{-m}\right|>\left|\xi_{0}^{-1} \sum_{j=0}^{m-1} \xi_{0}^{-(m-j-1)} \sum_{k=0}^{n} c_{j k} \eta^{k}\right|
$$

on $|\eta-\nu|=\delta$. Then by virtue of the theorem of Rouché, $P\left(\xi_{0}, \eta\right)-\lambda$ has at least a zero in $|\eta-\nu|<\delta$, a contradiction.

(b) We show only for $P\{A \otimes I, I \otimes B\}$. For $A$ bounded it is trivial. If $A$ is unbounded then by the proof of (a), $\sum_{k=0}^{n} c_{m k} \eta^{k}$ does not vanish on $\sigma(B)$, so that, by Proposition 1.2, $\sum_{k=0}^{n} c_{m k} B^{k}$ has an everywhere defined bounded inverse in $Y$. Then the assertion can be shown by induction on $t$.

(c) $B$ is bounded with $D[B]=Y$. Proving the first equality is equivalent to proving the closedness of $(0.2)$. For $A$ bounded it is obvious. Therefore assume $A$ is unbounded. Let $\left\{u_{l}\right\}_{l=1}^{\infty}$ be in $D[P\{A \otimes I, I \otimes B\}]=D\left[A^{m}\right]$ $\otimes Y$ and let $u_{l} \rightarrow u, P\{A \otimes I, I \otimes B\} u_{l} \rightarrow v$ in $X \otimes Y$ in the norm $\alpha$ as $l \rightarrow \infty$. Then by continuity we obtain for $\mu \in \rho(A)$

$$
\sum_{j=0}^{m}\left[A^{j}(A-\mu I)^{-m} \otimes\left(\sum_{k=0}^{n} c_{j k} B^{k}\right)\right] u=\left[(A-\mu I)^{-m} \otimes I\right] v .
$$

$u$ has a representation $u=\sum_{i=1}^{r} x_{i} \otimes y_{i}$, where both the sequences $\left\{x_{i}\right\}_{i=1}^{r} \subset$ $X$ and $\left\{y_{i}\right\}_{i=1}^{r} \subset Y$ are linearly independent. Since $\sum_{k=0}^{n} c_{m k} B^{k}$ is one-to-one as seen in the proof of (a), we see by (b) that for $1 \leqslant p \leqslant r$

$$
A^{m}(A-\mu I)^{-m} x_{p}+\sum_{i=1}^{r} \sum_{j=0}^{m-1} c_{j} A^{j}(A-\mu I)^{-m} x_{i}
$$

is in $D\left[A^{m}\right]$, where $c_{j}$ are certain constants. Hence $\left\{x_{i}\right\}_{i=1}^{r} \subset D\left[A^{m}\right]$ and $u \in D\left[A^{m}\right] \otimes Y$. Thus $(0.2)$ is closed.

To establish the second equality we have only to show the domain of $P\{A \otimes I, I \otimes B\}^{\prime}$ is included in $D\left[\left(A^{\prime}\right)^{m}\right] \otimes Y^{\prime}$. Let $u^{\prime}$ be in

$$
D\left[P\{A \otimes I, I \otimes B\}^{\prime}\right], \quad u^{\prime}=\sum_{i=1}^{r} x_{i}^{\prime} \otimes y_{i}^{\prime},
$$

where both the sequences $\left\{x_{i}^{\prime}\right\}_{i=1}^{r} \subset X^{\prime}$ and $\left\{y_{i}^{\prime}\right\}_{i=1}^{r} \subset Y^{\prime}$ are linearly independent. Since $\sum_{k=0}^{n} c_{m k} B^{k}$ maps onto $Y$ there exists a sequence $\left\{y_{i}\right\}_{i=1}^{r} \subset Y$ with $\left\langle\sum_{k=0}^{n} c_{m k} B^{k} y_{p}, y_{q}^{\prime}\right\rangle=\delta_{p q}, p, q=1,2, \ldots, r$. Then for all $x \in D\left[A^{m}\right]$ and for $1 \leqslant p \leqslant r$ we have

$$
\begin{gathered}
\left\langle A^{m} x, x_{p}^{\prime}\right\rangle+\sum_{i=1}^{r} \sum_{j=0}^{m-1}\left\langle A^{j} x, x_{i}^{\prime}\right\rangle\left\langle\sum_{k=0}^{n} c_{j k} B^{k} y_{p}, y_{i}^{\prime}\right\rangle \\
=\left\langle P\{A \otimes I, I \otimes B\}\left(x \otimes y_{p}\right), u^{\prime}\right\rangle \\
=\left\langle x \otimes y_{p}, P\{A \otimes I, I \otimes B\}^{\prime} u^{\prime}\right\rangle .
\end{gathered}
$$


Hence it is easy to see inductively that $\left\{x_{i}^{\prime}\right\}_{i=1}^{r} \subset D\left[\left(A^{\prime}\right)^{m}\right]$, whence $u^{\prime} \in$ $D\left[\left(A^{\prime}\right)^{m}\right] \otimes Y^{\prime}$.

(d) We show only the first inequality. By (b) and (c), both $A^{\text {lm }} \otimes I$ and $P\{A \otimes I, I \otimes B\}^{t}$ are closed in the Banach space $X \otimes Y$ equipped with the norm $\alpha$ and have the same domain. It is easy to verify that there is a constant $C_{1}$ such that for all $u \in D\left[A^{t m}\right] \otimes Y$

$$
\left\|P\{A \otimes I, I \otimes B\}^{t} u\right\|_{\alpha}<C_{1}\left[\left\|\left[A^{t m} \otimes I\right] u\right\|_{\alpha}+\|u\|_{\alpha}\right] .
$$

Here recall that all reasonable norms on $X \otimes Y$ are equivalent, because $Y$ is of finite dimension. Then by virtue of the closed graph theorem, the graph norm of $P\{A \otimes I, I \otimes B\}^{t}$ is equivalent to that of $A^{t m} \otimes I$. Since $P_{\lambda}(\xi, \nu)=$ $P(\xi, \nu)-\lambda$ is of degree $<m$ in $\xi$, the graph norm of $P_{\lambda}(A, \nu)^{t} \otimes I$ is majorized by that of $A^{t m} \otimes I$ and hence by that of $P\{A \otimes I, I \otimes B\}^{t}$. Q.E.D.

Remark. From the proof it is seen that the assertions (a) and (b) in Proposition 2.1 are valid for both $X$ and $Y$ of infinite dimension.

The proofs of the theorems in the next section will depend on examining the properties of the parts of $\mathbf{P}$ in its suitable invariant subspaces.

Associated with each finite subset $\left\{\alpha_{j}\right\}_{j=1}^{a}$ of $\sigma(A) \backslash \sigma_{e b}(A)$ is the projection $P$ in $X$ :

$$
P=(2 \pi i)^{-1} \sum_{j=1}^{a} \int_{C_{j}}(\xi I-A)^{-1} d \xi .
$$

Here $C_{j}, 1<j<a$, are disjoint circles round $\alpha_{j}$ such that $\alpha_{j}$ is the only point of $\sigma(A)$ contained in $C_{j}$. In the same way, associated with each finite subset $\left\{\beta_{k}\right\}_{k=1}^{b}$ of $\sigma(B) \backslash \sigma_{e b}(B)$ is the projection $Q$ in $Y . P$ and $Q$ are finite-dimensional and commute with $A$ and $B$, respectively, i.e. $P A \subset A P, Q B \subset B Q$. By Proposition 1.5,

$$
\begin{aligned}
X \hat{\otimes}_{\alpha} Y=R\left[P \hat{\otimes}_{\alpha} Q\right] \oplus R\left[P \hat{\otimes}_{\alpha}(I-Q)\right] \\
\\
\oplus R\left[(I-P) \hat{\otimes}_{\alpha} Q\right] \oplus R\left[(I-P) \hat{\otimes}_{\alpha}(I-Q)\right],
\end{aligned}
$$

where

$$
\begin{aligned}
& R\left[P \hat{\otimes}_{\alpha} Q\right]=P X \otimes Q Y, \\
& R\left[P \hat{\otimes}_{\alpha}(I-Q)\right]=P X \hat{\otimes}_{\bar{\alpha}}(I-Q) Y=P X \otimes(I-Q) Y, \\
& R\left[(I-P) \hat{\otimes}_{\alpha} Q\right]=(I-P) X \hat{\otimes}_{\bar{\alpha}} Q Y=(I-P) X \otimes Q Y, \\
& R\left[(I-P) \hat{\otimes}_{\alpha}(I-Q)\right]=(I-P) X \hat{\otimes}_{\bar{\alpha}}(I-Q) Y .
\end{aligned}
$$

The projections $P \hat{\otimes}_{\alpha} Q, P \hat{\otimes}_{\alpha}(I-Q),(I-P) \hat{\otimes}_{\alpha} Q$ and $(I-P) \hat{\otimes}_{\alpha}(I$ $-Q$ ) commute with $\mathbf{P}_{\lambda}$. Consequently $\mathbf{P}_{\lambda}$ is decomposed by these four 
subspaces of $X \hat{\otimes}_{\alpha} Y$ in (2.3). Its parts in the respective subspaces in (2.3) are denoted by $\mathbf{P}_{\lambda 11}, \mathbf{P}_{\lambda 12}, \mathbf{P}_{\lambda 21}$ and $\mathbf{P}_{\lambda 22}$; for $\lambda=0$ set $\mathbf{P}_{j k}=\mathbf{P}_{0 j k}, j, k=1,2$.

The parts of $A$ in $P X,(I-P) X$ are denoted by $A_{1}, A_{2}$ and the parts of $B$ in $Q Y,(I-Q) Y$ by $B_{1}, B_{2}$. Then $A_{1}$ and $B_{1}$ are bounded on $P X, Q Y$, respectively, with $\sigma\left(A_{1}\right)=\left\{\alpha_{j}\right\}_{j=1}^{a}, \sigma\left(B_{1}\right)=\left\{\beta_{k}\right\}_{k=1}^{b}$, while $A_{2}$ and $B_{2}$ are closed in $(I-P) X,(I-Q) Y$, respectively, with spectra

$$
\sigma\left(A_{2}\right)=\sigma(A) \backslash\left\{\alpha_{j}\right\}_{j=1}^{a}, \quad \sigma\left(B_{2}\right)=\sigma(B) \backslash\left\{\beta_{k}\right\}_{k=1}^{b} .
$$

The identity operators in $P X, Q Y$ (resp. $(I-P) X,(I-Q) Y)$ are denoted by the same $I_{1}$ (resp. $I_{2}$ ).

Proposition 2.3. If $P(\xi, \eta)$ is in $\mathscr{P}_{e}(A, B)$ then it is also in $\mathscr{P}_{e}\left(A_{j}, B_{k}\right)$ for $j, k=1,2$.

Proof. Let $P \in \mathscr{P}_{e}(A, B)$. Since $A_{1}$ and $B_{1}$ are bounded every polynomial belongs to $\mathcal{P}_{e}\left(A_{1}, B_{1}\right)$. The proofs of the other three cases are analogous and so we show only the case $j=k=2$.

We may assume both $\sigma\left(A_{2}\right)$ and $\sigma\left(B_{2}\right)$ nonempty. Let $\lambda \notin P\left(\sigma\left(A_{2}\right), \sigma\left(B_{2}\right)\right)$ with dist $\left(\lambda, P\left(\sigma\left(A_{2}\right), \sigma\left(B_{2}\right)\right)\right)>0$. We may assume $\lambda$ is in $P(\sigma(A), \sigma(B))$; otherwise the assertion is obvious. We must find nonempty open sets $U_{2}$ and $V_{2}$ with $C U_{2} \subset \rho\left(A_{2}\right)$ and $C V_{2} \subset \rho\left(B_{2}\right)$ having the properties with $(I-P) X$, $(I-Q) Y, A_{2}, B_{2}, U_{2}, V_{2}$ and $\lambda$ in place of $X, Y, A, B, U, V$ and $\kappa$, respectively, in definition of $\mathscr{P}_{e}(A, B)$.

Since $P \in \mathscr{P}_{e}(A, B)$ we can choose, for some fixed $k \notin P(\sigma(A), \sigma(B))$, nonempty open sets $U, V$ with $C U \subset \rho(A), C V \subset \rho(B)$ having the properties mentioned in definition of $\mathscr{P}_{e}(A, B)$ and a large $r>0$ such that both $\sigma\left(A_{2}\right) \cap K_{r}$ and $\sigma\left(B_{2}\right) \cap K_{r}$ are nonempty and such that

$$
|P(\xi, \eta)| \geqslant|\lambda|+1 \quad \text { on } \bar{U}+\bar{V}, \quad|\xi|+|\eta|>r \text {. }
$$

Then if $U \cap \mathrm{C} K_{r}$ (resp. $V \cap \mathrm{C} K_{r}$ ) is nonempty,

$$
\operatorname{dist}\left(\lambda, P\left(U \cap C K_{r}, V\right)\right)>1 \quad\left(\text { resp. } \operatorname{dist}\left(\lambda, P\left(U, V \cap C K_{r}\right)\right)>1\right) .
$$

Since $\sigma\left(A_{2}\right) \cap K_{r}$ and $\sigma\left(B_{2}\right) \cap K_{r}$ are compact, there exist nonempty, bounded open sets $U_{1}$ and $V_{1}$ with boundaries consisting of a finite number of rectifiable Jordan curves such that

$$
\begin{gathered}
\sigma\left(A_{2}\right) \cap K_{r} \subset U_{1} \subset U, \quad \sigma\left(B_{2}\right) \cap K_{r} \subset V_{1} \subset V, \\
\operatorname{dist}\left(\lambda, P\left(U_{1}, V_{1}\right)\right)>0 .
\end{gathered}
$$

Set $U_{2}=\left(U \cap \mathrm{C} K_{r}\right) \cup\left(U_{1} \cap K_{r}\right), V_{2}=\left(V \cap \mathrm{C} K_{r}\right) \cup\left(V_{1} \cap K_{r}\right)$. It is clear that $U_{2}$ and $V_{2}$ are the desired open sets. Q.E.D.

The closability of $P\{A \otimes I, I \otimes B\}$ implies the closability of $P\left\{A_{j} \otimes\right.$ $\left.I_{k}, I_{j} \otimes B_{k}\right\}$ for $j, k=1,2$. As a consequence of Proposition 2.3 we see that if 
$P \in \mathscr{P}_{e}(A, B)$ then Theorem 2.1 is valid with $A_{j}$ and $B_{k}, j, k=1,2$, in place of $A$ and $B$, respectively.

Proposition 2.4. If $P \in \mathcal{P}_{e}(A, B)$ then

$$
\begin{aligned}
& \mathbf{P}_{11}=P\left\{A_{1} \otimes I_{1}, I_{1} \otimes B_{1}\right\}, \\
& \mathbf{P}_{12}=P\left\{A_{1} \otimes I_{2}, I_{1} \otimes B_{2}\right\}, \\
& \mathbf{P}_{21}=P\left\{A_{2} \otimes I_{1}, I_{2} \otimes B_{1}\right\}, \\
& \mathbf{P}_{22}=\tilde{P}\left\{A_{2} \otimes I_{2}, I_{2} \otimes B_{2}\right\} .
\end{aligned}
$$

Proof. (2.5) is obvious. If $\lambda \notin P(\sigma(A), \sigma(B))$, by Theorem $2.1 \mathbf{P}_{\lambda}$ has an everywhere defined bounded inverse. Then the same is true of the parts $\mathbf{P}_{\lambda 12}$, $\mathbf{P}_{\lambda 21}$ and $\mathbf{P}_{\lambda 22}$. They are extensions of the operators on the right of (2.6)-(2.8). Therefore it suffices to show that the operators on the right of $(2.6)-(2.8)$ are closed and that all their resolvent sets contain $\lambda$. However, it is readily seen from Proposition 2.2 and Theorem 2.1 with Proposition 2.3 and from the fact that the operator on the right of (2.8) is the closure of $P\left\{A_{2} \otimes I_{2}, I_{2} \otimes B_{2}\right\}$. Q.E.D.

Throughout this paper, each of the parts of $\mathbf{P}_{\lambda}$, that is, $\mathbf{P}_{\lambda 11}, \mathbf{P}_{\lambda 12}, \mathbf{P}_{\lambda 21}$ and $\mathbf{P}_{\lambda 22}$, will be referred to as the part of $\mathbf{P}_{\lambda}$ relative to the sets $\left\{\alpha_{j}\right\}_{j=1}^{a}$ and $\left\{\beta_{k}\right\}_{k=1}^{b}$.

Finally we introduce several subsets of $\sigma(A) \times \sigma(B)$ :

$$
\Delta_{0}(\lambda)=\{(\xi, \eta) \in \sigma(A) \times \sigma(B) ; P(\xi, \eta)=\lambda\}
$$$$
\text { (2.10) } \Delta_{1}(\lambda)=\left\{(\xi, \eta) \in\left(\sigma(A) \backslash \sigma_{e b}(A)\right) \times\left(\sigma(B) \backslash \sigma_{e b}(B)\right) ; P(\xi, \eta)=\lambda\right\} \text {, }
$$$$
\text { (2.11) } \Delta_{10}(\lambda)=\left\{(\xi, \eta) \in\left(\sigma(A) \backslash \sigma_{e b}(A)\right) \times\left(\sigma(B) \backslash \sigma_{e w}(B)\right) ; P(\xi, \eta)=\lambda\right\},
$$$$
(2.12) \Delta_{01}(\lambda)=\left\{(\xi, \eta) \in\left(\sigma(A) \backslash \sigma_{e w}(A)\right) \times\left(\sigma(B) \backslash \sigma_{e b}(B)\right) ; P(\xi, \eta)=\lambda\right\} \text {, }
$$$$
\text { (2.13) } \Delta_{12}(\lambda)=\left\{(\xi, \eta) \in\left(\sigma(A) \backslash \sigma_{e b}(A)\right) \times\left(\sigma_{e b}(B) \backslash \sigma_{e w}(B)\right) ; P(\xi, \eta)=\lambda\right\} \text {, }
$$$$
(2.14) \Delta_{21}(\lambda)=\left\{(\xi, \eta) \in\left(\sigma_{e b}(A) \backslash \sigma_{e w}(A)\right) \times\left(\sigma(B) \backslash \sigma_{e b}(B)\right) ; P(\xi, \eta)=\lambda\right\} \text {. }
$$

3. Essential spectra for tensor products of linear operators. For the closed linear operator $\tilde{P}\{A \otimes I, I \otimes B\}$ in $X \hat{\otimes}_{\alpha} Y$, we shall first give exact representations of its Browder and Wolf essential spectra in terms of the parts of the spectra of $A$ and $B$, next derive formulae for its nullity, deficiency and index, and finally determine its Schechter essential spectrum with this very index.

We follow the same conventions as in $\$ 2$.

3.1. The Browder essential spectrum.

THEOREM 3.1. Let $\alpha$ be a quasi-uniform reasonable norm on $X \otimes Y$ and let $P \in \mathcal{P}_{e}(A, B)$. Then 


$$
\sigma_{e b}(\tilde{P}\{A \otimes I, I \otimes B\})=P\left(\sigma_{e b}(A), \sigma(B)\right) \cup P\left(\sigma(A), \sigma_{e b}(B)\right) .
$$

By this is meant that (3.1) holds valid if both $\sigma_{e b}(A)$ and $\sigma(B)$ are nonempty or if both $\sigma(A)$ and $\sigma_{e b}(B)$ are nonempty, and further that $\sigma_{e b}(\tilde{P}\{A \otimes I, I \otimes B\})$ is empty if and only if either $\sigma(A)=\varnothing$ or $\sigma(B)=\varnothing$ or $\sigma_{e b}(A)=\sigma_{e b}(B)=\varnothing$.

As will be seen from the proof of Theorem 3.1, the inclusion

$$
\sigma_{e b}(\tilde{P}\{A \otimes I, I \otimes B\}) \supset P\left(\sigma_{e b}(A), \sigma(B)\right) \cup P\left(\sigma(A), \sigma_{e b}(B)\right)
$$

holds valid in fact for every polynomial $P(\xi, \eta)$. To prove (3.2) we need Lemma 3.2 below. To prove the reverse inclusion we simply modify the method of R. D. Nussbaum [18] to fit the setting of tensor products.

LeMma 3.2. Let $P(\xi, \eta)$ be a polynomial $(0.1)$.

(a) If both $\operatorname{nul}^{\prime}(A-\mu I)$ and $\operatorname{nul}^{\prime}(B-\nu I)$ are positive and if at least one of them is infinite, then nul' $\mathbf{P}_{\lambda}$ is infinite with $\lambda=P(\mu, \nu)$.

(b) If both $\operatorname{def}^{\prime}(A-\mu I)$ and $\operatorname{def}^{\prime}(B-\nu I)$ are positive and if at least one of them is infinite, then $\operatorname{def}^{\prime} \mathbf{P}_{\lambda}$ is infinite with $\lambda=P(\mu, \nu)$.

(c) Let $P \in \mathscr{P}_{e}(A, B)$. If both $\operatorname{nul}^{\prime}(P(A, \nu)-\lambda I)$ and $\operatorname{nul}^{\prime}(B-\nu I)$ are positive and if at least one of them is infinite, then nul' $\mathbf{P}_{\lambda}$ is infinite.

(d) Let $P \in \mathcal{P}_{e}(A, B)$. If both $\operatorname{def}^{\prime}(P(A, \nu)-\lambda I)$ and $\operatorname{def}^{\prime}(B-\nu I)$ are positive and if at least one of them is infinite, then $\operatorname{def}^{\prime} \mathbf{P}_{\lambda}$ is infinite.

Proof. First note

$$
P(\xi, \eta)-P(\mu, \nu)=\sum_{j k} b_{j k}(\xi-\mu)^{j}(\eta-\nu)^{k}, \quad b_{00}=0,
$$

and

$$
\begin{aligned}
P(\xi, \eta)-\lambda & =(P(\xi, \eta)-P(\xi, \nu))+(P(\xi, \nu)-\lambda) \\
& =\sum_{k=1}^{n} \sum_{j=0}^{m} a_{j k} \xi^{j}(\eta-\nu)^{k}+(P(\xi, \nu)-\lambda) .
\end{aligned}
$$

(a) By the hypothesis with Lemma 1.3 there exist sequences of unit vectors, $\left\{x_{l}\right\}_{l=1}^{\infty} \subset D\left[A^{m}\right]$ and $\left\{y_{l}\right\}_{l=1}^{\infty} \subset D\left[B^{n}\right]$, such that $A^{j}(A-\mu I) x_{l} \rightarrow 0$ as $l \rightarrow \infty$ for $0 \leqslant j \leqslant m-1$ and $B^{k}(B-\nu I) y_{l} \rightarrow 0$ as $l \rightarrow \infty$ for $0<k<n-$ 1. Since either $\operatorname{nul}^{\prime}(A-\mu I)$ or $\operatorname{nul}^{\prime}(B-\nu I)$ is infinite, by Lemma 1.3 either $\left\{x_{l}\right\}_{l=1}^{\infty}$ or $\left\{y_{l}\right\}_{l=1}^{\infty}$ can be chosen to be noncompact. Then $\left\{x_{l} \otimes y_{l}\right\}_{l=1}^{\infty}$ is noncompact in $X \hat{\otimes}_{\varepsilon} Y$ (e.g. [9, Theorem 1]) and hence in $X \hat{\otimes}_{\alpha} Y$ because $\varepsilon \leqslant \alpha$. It is a sequence of unit vectors in $X \hat{\otimes}_{\alpha} Y$, since $\alpha$ is reasonable. By (3.3) we obtain with $\lambda=P(\mu, \nu)$

$$
\begin{aligned}
\mathbf{P}_{\lambda}\left(x_{l} \otimes y_{l}\right) & =[P\{A \otimes I, I \otimes B\}-P(\mu, \nu) I \otimes I]\left(x_{1} \otimes y_{1}\right), \\
& =\sum_{j+k>0} b_{j k}(A-\mu I)^{j} x_{l} \otimes(B-\nu I)^{k} y_{l},
\end{aligned}
$$


which approaches 0 in $X \hat{\otimes}_{\alpha} Y$ as $l \rightarrow \infty$. Hence nul' $\mathbf{P}_{\lambda}=\infty$.

(b) In this case, $\operatorname{nul}^{\prime}\left(A^{\prime}-\mu I^{\prime}\right)$ and $\operatorname{nul}^{\prime}\left(B^{\prime}-\nu I^{\prime}\right)$ are positive and one of them is infinite. By the same argument as in (a) the approximate nullity of the closed linear operator

$$
\tilde{P}\left\{A^{\prime} \otimes I^{\prime}, I^{\prime} \otimes B^{\prime}\right\}-P(\mu, \nu) I^{\prime} \hat{\otimes}_{\alpha^{\prime}} I^{\prime}
$$

in $X^{\prime} \hat{\otimes}_{\alpha^{\prime}} Y^{\prime}$ is infinite. Since $P_{\lambda}^{\prime}$ with $\lambda=P(\mu, \nu)$ is an extension of (3.5), both as closed operators in the dual space $\left(X \hat{\otimes}_{\alpha} Y\right)^{\prime}$, we obtain def' $\mathbf{P}_{\lambda}=$ nul' $^{\prime} \mathbf{P}_{\lambda}^{\prime}=\infty$ with $\lambda=P(\mu, \nu)$.

(c) By the hypothesis with Lemma 1.3 we can choose a sequence $\left\{y_{l}\right\}_{l=1}^{\infty} \subset$ $D\left[B^{n}\right]$ of unit vectors with $B^{k}(B-\nu I) y_{l} \rightarrow 0$ as $l \rightarrow \infty$ for $0<k<n-1$. Note that by Proposition 2.2(a) with its Remark $D[P(A, v)]=D\left[A^{m}\right]$ for $\nu \in \sigma(B)$ and choose a sequence $\left\{x_{l}\right\}_{l=1}^{\infty} \subset D\left[A^{m}\right]$ of unit vectors with $(P(A, \nu)-\lambda I) x_{l} \rightarrow 0$ as $l \rightarrow \infty$. Since the graph norm of $P(A, \nu)$ is equivalent to the norm (1.7) with $T=A$, each sequence $\left\{A^{j} x_{l}\right\}_{l=1}^{\infty}, 0<j<m$, is bounded. By hypothesis either $\left\{x_{l}\right\}_{l=1}^{\infty}$ or $\left\{y_{l}\right\}_{l=1}^{\infty}$ can be chosen to be noncompact. Then an argument analogous to (a) using (3.4) will yield the desired result.

(d) The hypothesis implies that both

$$
\operatorname{nul}^{\prime}\left(P\left(A^{\prime}, \nu\right)-\lambda I^{\prime}\right) \text { and } \operatorname{nul}^{\prime}\left(B^{\prime}-\nu I^{\prime}\right)
$$

are positive and one of them is infinite. The same argument as in (c) and (b) will yield the result. Q.E.D.

Proof of Theorem 3.1. (a) Proof of the Inclusion (3.2). Because of symmetry it suffices to show $P\left(\sigma_{e b}(A), \sigma(B)\right) \subset \sigma_{e b}(\mathrm{P})$, assuming $\sigma_{e b}(A)$ and $\sigma(B)$ are nonempty.

Let $\lambda \in P\left(\sigma_{e b}(A), \sigma(B)\right)$. By Theorem 2.1, $\lambda$ belongs to $\sigma(\mathrm{P})$. We want to show $\lambda \in \sigma_{e b}(\mathbf{P})$. Assume not, so that $\lambda$ is an isolated, finite-dimensional eigenvalue of $\mathrm{P}$. So $\lambda$ is an isolated point of $\sigma(\mathrm{P})$ and hence $P\left(\sigma_{e b}(A), \sigma(B)\right)$. Both $\sigma_{e b}(A)$ and $\sigma(B)$ are nonempty, closed proper subsets of $C$. It is then easy to see that there are boundary points $\xi_{0}$ of $\sigma_{e b}(A)$ and $\eta_{0}$ of $\sigma(B)$ with $P\left(\xi_{0}, \eta_{0}\right)=\lambda$. By Proposition 1.1, $\xi_{0} \in \sigma_{e k}(A)$ and $\eta_{0} \in \sigma_{\pi}(B)$, so that

$$
\operatorname{nul}^{\prime}\left(A-\xi_{0} I\right)=\infty \text { and } \operatorname{nul}^{\prime}\left(B-\eta_{0} I\right)>0 \text {. }
$$

Then by Lemma 3.2(a) nul' $\mathbf{P}_{\lambda}=\infty$. Since the range $R\left[\mathbf{P}_{\lambda}\right]$ of $\mathbf{P}_{\lambda}$ is closed it follows that nul $\mathbf{P}_{\lambda}=\infty$, that is, $\lambda$ is an infinite-dimensional eigenvalue of $\mathbf{P}$, contrary to assumption. This proves $\lambda \in \sigma_{e b}(P)$.

(b) Proof of the Reverse inclusion. Let $\lambda \in \sigma_{e b}(P)$. Since $P \in \mathcal{P}_{e}(A, B)$ there are nonempty open sets $U \supset \sigma(A), V \supset \sigma(B)$ and a large $r>0$ such that (2.4) holds. For each small $\varepsilon>0$, set

$$
U_{\varepsilon}=\left\{\zeta ; \operatorname{dist}\left(\zeta, \sigma_{e b}(A) \cap K_{r}\right)<\varepsilon\right\} \cup\left(U \cap C K_{r}\right)
$$


and

$$
V_{e}=\left\{\zeta ; \operatorname{dist}\left(\zeta, \sigma_{e b}(B) \cap K_{r}\right)<\varepsilon\right\} \cup\left(V \cap \mathrm{C} K_{r}\right),
$$

if both $\sigma_{e b}(A) \cap K_{r}$ and $\sigma_{e b}(B) \cap K_{r}$ are nonempty, while if $\sigma_{e b}(A) \cap K_{r}=\varnothing$ (resp. $\sigma_{e b}(B) \cap K_{r}=\varnothing$ ), set $U_{\varepsilon}=U \cap \mathrm{C} K_{r}$ (resp. $V_{\varepsilon}=V \cap \mathrm{C} K_{r}$ ). If $\varepsilon$ is sufficiently small $U_{\varepsilon}$ is included in $U$ and $V_{\varepsilon}$ in $V$.

To prove that $\lambda$ belongs to the right member of (3.1) it suffices to show that, for every sufficiently small $\varepsilon>0, \lambda$ belongs to $P\left(U_{e}, \sigma(B)\right) \cup$ $P\left(\sigma(A), V_{\varepsilon}\right)$. In fact, suppose this is established. For each sufficiently large integer $l$ choose $\left(\xi_{l}, \eta_{l}\right)$ with $P\left(\xi_{l}, \eta_{l}\right)=\lambda$ in

$$
\left\{\left(U_{1 / l} \cap K_{r}\right) \times\left(\sigma(B) \cap K_{r}\right)\right\} \cup\left\{\left(\sigma(A) \cap K_{r}\right) \times\left(V_{1 / l} \cap K_{r}\right)\right\} .
$$

Since $\left\{\left(\xi_{l}, \eta_{l}\right)\right\}_{l=1}^{\infty}$ is bounded, we may assume, by taking subsequences, that it is convergent to $\left(\xi_{0}, \eta_{0}\right)$ as $l \rightarrow \infty$. Obviously $\left(\xi_{0}, \eta_{0}\right)$ belongs to $\left(\sigma_{e b}(A) \times\right.$ $\sigma(B)) \cup\left(\sigma(A) \times \sigma_{e b}(B)\right)$ and $P\left(\xi_{0}, \eta_{0}\right)=\lambda$, whence $\lambda$ belongs to the right member of (3.1).

If $\sigma(A) \subset U_{\varepsilon}$ or $\sigma(B) \subset V_{\varepsilon}$ then the desired assertion is evident by Theorem 2.1. Otherwise both the sets

$$
\sigma(A) \backslash U_{\varepsilon}=\left\{\alpha_{j}\right\}_{j=1}^{a} \text { and } \sigma(B) \backslash V_{e}=\left\{\beta_{k}\right\}_{k=1}^{b}
$$

are nonempty and finite. Let $P$ and $Q$ be the projections associated with $\left\{\alpha_{j}\right\}_{j=1}^{a}$ and $\left\{\beta_{k}\right\}_{k=1}^{b}$, respectively.

In view of (2.4) choose $\left(\alpha_{0}, \beta_{0}\right) \in U \times V$ such that $\left|P\left(\alpha_{0}, \beta_{0}\right)\right|>|\lambda|+1$. Consider the operator

$$
\begin{aligned}
P\{A \otimes I & \left.+A_{0} \otimes Q, I \otimes B+P \otimes B_{0}\right\} \\
& \equiv \sum_{j k} c_{j k} \sum_{s=0}^{j} \sum_{t=0}^{k}\left(\begin{array}{l}
j \\
s
\end{array}\right)\left(\begin{array}{l}
k \\
t
\end{array}\right) A_{0}^{s} P^{t} A^{j-s} \otimes Q^{s} B_{0}^{t} B^{k-t}
\end{aligned}
$$

in $X \hat{\otimes}_{\alpha} Y$ with domain $D\left[A^{m}\right] \otimes D\left[B^{n}\right]$, where $A_{0}=\alpha_{0} P-A P$ and $B_{0}=$ $\beta_{0} Q-B Q$ are bounded linear operators of finite rank on $X, Y$ which commute with $A, B$, respectively. It is easy to check that there exists a bounded linear operator $\mathbf{R}$ on $X \hat{\otimes}_{\alpha} Y$ of finite rank which commutes with (0.2) and such that for $u \in D\left[A^{m}\right] \otimes D\left[B^{n}\right]$,

$$
P\left\{A \otimes I+A_{0} \otimes Q, I \otimes B+P \otimes B_{0}\right\} u-P\{A \otimes I, I \otimes B\} u=\mathbf{R} u \text {. }
$$

Since (0.2) is closable, (3.6) is closable; we denote the closure of (3.6) by $\mathbf{Q}$. Then $D[\mathbf{Q}]=D[\mathbf{P}]$. $\mathbf{R}$ commutes with $\mathbf{P}$. For $u \in D[\mathbf{P}]$ we have $\mathbf{Q} u-\mathbf{P} u=$ $\mathbf{R} u . \mathbf{Q}$ is decomposed by the four subspaces of $X \hat{\otimes}_{\alpha} Y$ in (2.3). Its parts in the respective subspaces are

$$
\mathbf{Q}_{11}=P\left(\alpha_{0}, \beta_{0}\right)\left(I_{1} \otimes I_{1}\right), \quad \mathbf{Q}_{12}=\mathbf{P}_{12}, \quad \mathbf{Q}_{21}=\mathbf{P}_{21} \quad \text { and } \quad \mathbf{Q}_{22}=\mathbf{P}_{22} \text {. }
$$

Since the Browder essential spectrum of a closed operator $T$ remains 
invariant under perturbations of $T$ by compact operators commuting with $T$ ([16], [13]), we obtain

$$
\sigma_{e b}(\mathbf{P})=\sigma_{e b}(\mathbf{Q}) \subset \sigma(\mathbf{Q})=\sigma\left(\mathbf{Q}_{11}\right) \cup \sigma\left(\mathbf{Q}_{12}\right) \cup \sigma\left(\mathbf{Q}_{21}\right) \cup \sigma\left(\mathbf{Q}_{22}\right) .
$$

It is easy to verify by Theorem 2.1 with Propositions 2.3 and 2.4

$$
\begin{array}{lll}
\sigma\left(\mathbf{Q}_{11}\right)=\left\{P\left(\alpha_{0}, \beta_{0}\right)\right\}, & \sigma\left(\mathbf{Q}_{12}\right)=P\left(\sigma\left(A_{1}\right), \sigma\left(B_{2}\right)\right), \\
\sigma\left(\mathbf{Q}_{21}\right)=P\left(\sigma\left(A_{2}\right), \sigma\left(B_{1}\right)\right), & \sigma\left(\mathbf{Q}_{22}\right)=P\left(\sigma\left(A_{2}\right), \sigma\left(B_{2}\right)\right) .
\end{array}
$$

It follows that $\sigma_{e b}(\mathbf{P})$ is included in

$$
\left\{P\left(\alpha_{0}, \beta_{0}\right)\right\} \cup P\left(\sigma\left(A_{1}\right), \sigma\left(B_{2}\right)\right) \cup P\left(\sigma\left(A_{2}\right), \sigma\left(B_{1}\right)\right) \cup P\left(\sigma\left(A_{2}\right), \sigma\left(B_{2}\right)\right) .
$$

Since $\lambda \neq P\left(\alpha_{0}, \beta_{0}\right), \lambda$ belongs to

$$
\begin{gathered}
P\left(\sigma(A) \backslash\left\{\alpha_{j}\right\}_{j=1}^{a}, \sigma(B)\right) \cup P\left(\sigma(A), \sigma(B) \backslash\left\{\beta_{k}\right\}_{k=1}^{b}\right) \\
\subset P\left(U_{\varepsilon}, \sigma(B)\right) \cup P\left(\sigma(A), V_{\varepsilon}\right) \text {. Q.E.D. }
\end{gathered}
$$

Theorems 2.1 and 3.1 determine the set of all isolated, finite-dimensional eigenvalues of $\tilde{P}\{A \otimes I, I \otimes B\}$.

COROLlary 3.3. The same hypothesis as in Theorem 3.1. Then

$$
\begin{aligned}
\sigma(\tilde{P}\{A \otimes I, I \otimes B\}) \backslash \sigma_{e b}(\tilde{P}\{A \otimes I, I \otimes B\}) \\
=P\left(\sigma(A) \backslash \sigma_{e b}(A), \sigma(B) \backslash \sigma_{e b}(B)\right) \\
\backslash\left\{P\left(\sigma_{e b}(A), \sigma(B)\right) \cup P\left(\sigma(A), \sigma_{e b}(B)\right)\right\} .
\end{aligned}
$$

For each $\lambda$ in the set (3.7), the set (2.10), $\Delta_{1}(\lambda)$, is finite, $\Delta_{0}(\lambda)=\Delta_{1}(\lambda)$ and

$$
t(\tilde{P}\{A \otimes I, I \otimes B\} ; \lambda)=\sum_{(\mu, \nu) \in \Delta_{1}(\lambda)} t(A ; \mu) t(B ; \nu),
$$

where $t(T ; \kappa)$ is the algebraic multiplicity of an isolated eigenvalue $\kappa$ of $T$.

PROOF. We shall be concerined only with the algebraic multiplicity of $\lambda$, because the other assertions are easy to see from definition of the Browder essential spectrum.

Denote the image of the projection of $\Delta_{1}(\lambda)$ into the $\xi$ (resp. $\eta$ ) coordinate by $\Delta_{1}(\lambda ; A)$ (resp. $\Delta_{1}(\lambda ; B)$ ). Then both of them are finite, for $\Delta_{1}(\lambda)$ is finite. Since $\mathbf{P}_{\lambda}$ is Fredholm, the parts $\mathbf{P}_{\lambda 11}, \mathbf{P}_{\lambda 12}, \mathbf{P}_{\lambda 21}$ and $\mathbf{P}_{\lambda 22}$ relative to $\Delta_{1}(\lambda ; A)$ and $\Delta_{1}(\lambda ; B)$ are Fredholm. In the same way as in the proof of Theorem 3.1 we obtain

$$
\begin{aligned}
& \sigma\left(\mathbf{P}_{11}\right)=P\left(\Delta_{1}(\lambda ; A), \Delta_{1}(\lambda ; B)\right), \\
& \sigma\left(\mathbf{P}_{12}\right)=P\left(\Delta_{1}(\lambda ; A), \sigma(B) \backslash \Delta_{1}(\lambda ; B)\right), \\
& \sigma\left(\mathbf{P}_{21}\right)=P\left(\sigma(A) \backslash \Delta_{1}(\lambda ; A), \Delta_{1}(\lambda ; B)\right), \\
& \sigma\left(\mathbf{P}_{22}\right)=P\left(\sigma(A) \backslash \Delta_{1}(\lambda ; A), \sigma(B) \backslash \Delta_{1}(\lambda ; B)\right) .
\end{aligned}
$$


By definition of $\Delta_{1}(\lambda), \lambda$ belongs to $\sigma\left(\mathbf{P}_{11}\right)$ but not to the other three sets. Consequently $t(\mathrm{P} ; \lambda)=t\left(\mathrm{P}_{11} ; \lambda\right)$. On the other hand, $t\left(\mathrm{P}_{11} ; \lambda\right)$ is equal to the dimension of the projection $\Sigma_{(\mu, \nu) \in \Delta_{1}(\lambda)} P_{\mu} \hat{\otimes}_{\alpha} Q_{\nu}$, for if $(\mu, \nu)$ is in $\Delta_{1}(\lambda ; A) \times$ $\Delta_{1}(\lambda ; B)$ with $P(\mu, \nu) \neq \lambda$ then the part of $P_{11}$ in $R\left[P_{\mu} \hat{\otimes}_{\alpha} Q_{\nu}\right]$ has the spectrum not containing $\lambda$. Hence follows the result. Here $P_{\mu}$ and $Q_{\nu}$ are the projections associated with $\mu$ and $\nu$, respectively. Q.E.D.

3.2. The Wolf essential spectrum.

THEOREM 3.4. Let $\alpha$ be a quasi-uniform reasonable norm on $X \otimes Y$ and let $P \in \mathscr{P}_{e}(A, B)$. Then

(3.9) $\sigma_{e w}(\tilde{P}\{A \otimes I, I \otimes B\})=P\left(\sigma_{e w}(A), \sigma(B)\right) \cup P\left(\sigma(A), \sigma_{e w}(B)\right)$.

By this is meant that (3.9) holds valid if both $\sigma_{e w}(A)$ and $\sigma(B)$ are nonempty or if both $\sigma(A)$ and $\sigma_{e w}(B)$ are nonempty, and further that $\sigma_{e w}(\tilde{P}\{A \otimes I, I \otimes B\})$ is empty if and only if either $\sigma(A)=\varnothing$ or $\sigma(B)=\varnothing$ or $\sigma_{e w}(A)=\sigma_{e w}(B)=\varnothing$.

It will be seen from the proof of Theorem 3.4 that the inclusion

$$
\begin{aligned}
\sigma_{e w}(\tilde{P}\{A \otimes I, I \otimes B\}) \supset P\left(\sigma_{e w}(A), \sigma(B)\right) \cup & P\left(\sigma(A), \sigma_{e w}(B)\right) \\
& \cup P\left(\sigma_{e b}(A), \sigma_{e b}(B)\right)
\end{aligned}
$$

holds valid in fact for every polynomial $P(\xi, \eta)$.

Proof of Theorem 3.4. (a) Proof of THE inclusion (3.10). First note the right member of (3.10) coincides with the right member of (3.9). In fact, by Proposition 1.1 both $\Phi_{A}=\sigma_{e b}(A) \backslash \sigma_{e w}(A)$ and $\Phi_{A}=\sigma_{e b}(B) \backslash \sigma_{e w}(B)$ are open in C. If both of them are nonempty, we have only to see that $P\left(\Phi_{A}, \Phi_{B}\right)$ is a subset of the right member of (3.9). Let $\lambda \in P\left(\Phi_{A}, \Phi_{B}\right)$. Then it is seen (e.g. [11, Lemma 3.7]) that there exists a boundary point $\left(\xi_{0}, \eta_{0}\right)$ of $\Phi_{A} \times \Phi_{B}$ $\subset C^{2}$ with $P\left(\xi_{0}, \eta_{0}\right)=\lambda$, which belongs to $\sigma_{e w}(A) \times \sigma(B)$ or $\sigma(A) \times \sigma_{e w}(B)$ by Proposition 1.1. Hence $\lambda=P\left(\xi_{0}, \eta_{0}\right)$ belongs to the right member of (3.9).

Thus, to prove (3.10) it suffices to show $P\left(\sigma_{e w}(A), \sigma(B)\right) \subset \sigma_{e w}(P)$.

Let $\lambda \in P\left(\sigma_{e w}(A), \sigma(B)\right)$. If $R\left[\mathbf{P}_{\lambda}\right]$ is not closed, $\lambda$ belongs to $\sigma_{e w}(\mathbf{P})$. In the sequel we may therefore assume it is closed. The proof relies greatly upon Lemma 3.2.

Let $(\mu, \nu) \in \sigma_{e w}(A) \times \sigma(B)$ with $P(\mu, \nu)=\lambda$. The proof is divided into six cases. Note $\nu \in \sigma(B)$ if and only if $\operatorname{nul}(B-\nu I)>0$ or $\operatorname{def}(B-\nu I)>0$.

It is easy to check that the hypothesis in (a) or (b) of Lemma 3.2 is satisfied in the following four cases:

I. $R[A-\mu I]$ is not closed and $\operatorname{nul}(B-\nu I)>0$;

II. $R[A-\mu I]$ is not closed and $\operatorname{def}(B-\nu I)>0$;

III. $R[A-\mu I]$ is closed, $\operatorname{nul}(A-\mu I)=\infty$ and $\operatorname{nul}(B-\nu I)>0$;

IV. $R[A-\mu I]$ is closed, $\operatorname{def}(A-\mu I)=\infty$ and $\operatorname{def}(B-\nu I)>0$; so that the desired assertion follows from Lemma 3.2. 
V. In case $R[A-\mu I]$ is closed, $\operatorname{nul}(A-\mu I)=\infty$ and $\operatorname{def}(B-\nu I)>0$, we may assume in view of the cases III and IV that $\operatorname{def}(A-\mu I)<\infty$ and $\operatorname{nul}(B-\nu I)=0$. If $R[B-\nu I]$ is not closed this case is reduced to the case I with $A$ and $B$ replaced by each other. Therefore assume $R[B-\nu I]$ is also closed. Then $\mu$ lies in the semi-Fredholm domain $\rho_{e k}(A)$ of $A$ and $\nu$ in the semi-Fredholm domain $\rho_{e k}(B)$ of $B$. By Proposition 1.1 both

$$
\Psi_{A}=\sigma_{e b}(A) \backslash \sigma_{e k}(A) \text { and } \Psi_{B}=\sigma_{e b}(A) \backslash \sigma_{e k}(B)
$$

are open in $C$. Then by [14, Theorem 6],

$$
\operatorname{nul}(B-\eta I)=0, \quad \operatorname{def}(B-\eta I)=\operatorname{def}(B-\nu I)>0
$$

in a neighbourhood of $\eta=\nu$ in $\Psi_{B}$. Further, $\operatorname{def}(B-\eta I)>\operatorname{def}(B-\nu I)>0$ in the component $\Psi_{B}(\nu)$ in $\Psi_{B}$ containing $\nu$. In the same way nul $(A-\xi I)=$ $\infty$ in the component $\Psi_{A}(\mu)$ in $\Psi_{A}$ containing $\mu$. Then by an analogous argument used previously, there is a boundary point $\left(\psi_{0}, \eta_{0}\right)$ of $\Psi_{A}(\mu) \times$ $\Psi_{B}(\nu) \subset C^{2}$ with $P\left(\xi_{0}, \eta_{0}\right)=P(\mu, \nu)=\lambda$, so that $\xi_{0} \in \sigma_{e k}(A)$ or $\eta_{0} \in \sigma_{e k}(B)$ by Proposition 1.1. If $\left(\xi_{0}, \eta_{0}\right)$ is in $\sigma_{e k}(A) \times \Psi_{B}(\nu)$ then $\operatorname{def}^{\prime}\left(A-\xi_{0} I\right)=\infty$ and $\operatorname{def}\left(B-\eta_{0} I\right)>0$. If it is in $\Psi_{A}(\mu) \times \sigma_{e k}(B)$ then

$$
\operatorname{nul}\left(A-\xi_{0} I\right)=\operatorname{nul}^{\prime}\left(B-\eta_{0} I\right)=\infty \text {. }
$$

Finally if it is in $\sigma_{e k}(A) \times \sigma_{e k}(B)$ then $\operatorname{nul}^{\prime}\left(A-\xi_{0} I\right)=\operatorname{nul}^{\prime}\left(B-\eta_{0} I\right)=\infty$. Thus anyway the assertion follows from Lemma 3.2 (a) and (b).

VI. In the remaining case that $R[A-\mu I]$ is closed, $\operatorname{def}(A-\mu I)=\infty$ and $\operatorname{nul}(B-\nu I)>0$, we may assume in view of Lemma 3.2 that $\operatorname{nul}(A-\mu I)<$ $\infty$ and $R[B-\nu I]$ is closed with $\operatorname{def}(B-\nu I)=0$. Then $\mu$ (resp. $\nu$ ) lies in the semi-Fredholm domain of $A$ (resp. $B$ ). By [14, Theorem 6]

$$
\operatorname{def}(B-\eta I)=0, \operatorname{nul}(B-\eta I)=\operatorname{nul}(B-\nu I)>0
$$

in a neighbourhood of $\eta=\nu$ in $\Psi_{B}$. Further $\operatorname{nul}(B-\eta I)>\operatorname{nul}(B-\nu I)>0$ in the component in $\Psi_{B}$ containing $\nu$ and $\operatorname{def}(A-\xi I)=\infty$ in the component in $\Psi_{A}$ containing $\mu$. Then the same argument using Lemma 3.2 as in the case $\mathrm{V}$ will yield the result.

(b) ProOF OF THE REVERSE INCLUSION.

I. The case in which one of $X$ and $Y$, say $Y$, is of finite dimension. In this case let us keep in mind Proposition 2.2 and $P=P\{A \otimes I, I \otimes B\}$, then (3.9) becomes

$$
\sigma_{\text {ew }}(P\{A \otimes I, I \otimes B\})=P\left(\sigma_{\text {ew }}(A), \sigma(B)\right) .
$$

$B$ is bounded with $D[B]=Y$ and the spectrum $\sigma(B)$ of $B$ consists of a finite number of eigenvalues. We have

$$
Y=\sum_{\nu \in \sigma(B)} \oplus Y_{v}, \quad Y_{v}=N\left[(B-\nu I)^{q(B ; v)}\right]
$$


$B$ is decomposed by the $Y_{v}$. The part of $B$ in $Y_{v}$ is the sum of $\nu I_{\nu}$ and a nilpotent operator in $Y_{p}$, where $I_{v}$ is the identity operator in $Y_{v}$. Then $X \otimes Y$ is decomposed into the topological direct sum

$$
X \otimes Y=\sum_{\nu \in \sigma(B)} \oplus\left(X \otimes Y_{\nu}\right)
$$

and $\mathbf{P}$ is decomposed by the $X \otimes Y_{\nu}$.

Let $\lambda \in \sigma_{e w}(P)$. By Theorem 3.1 there are points $\mu \in \sigma_{e b}(A)$ and $\nu \in \sigma(B)$ with $P(\mu, \nu)=\lambda$. By the reduction method we have only to show that $\lambda$ belongs to the right member of (3.9)', assuming that $\nu$ is the only eigenvalue of $B$ with algebraic multiplicity $t(B ; \nu) \equiv t=\operatorname{dim} Y$, so that $B-\nu I$ is nilpotent.

If $P(\xi, \nu)-\lambda \equiv 0$ then by Theorem 2.1, $\sigma(\mathbf{P})$ consists of only one point $\lambda$. Then taking a boundary point $\mu^{\prime}$ of $\sigma_{e b}(A)$ we have $\lambda=P\left(\mu^{\prime}, \nu\right)$.

Therefore we consider the case $P(\xi, \nu)-\lambda \neq 0$. Recall that $\lambda \in \sigma_{\text {ew }}(P)$ if and only if either nul' $\mathbf{P}_{\lambda}$ or $\operatorname{def}^{\prime} \mathbf{P}_{\lambda}$ is infinite.

In case nul' $\mathbf{P}_{\lambda}$ is infinite, by Lemma 1.3 with Proposition 2.2(b) there exists a noncompact sequence $\left\{u_{l}\right\}_{l=1}^{\infty}$ of unit vectors in $D\left[A^{t m}\right] \otimes Y$ such that for $1<p<t$,

$$
\mathbf{P}^{p-1} \mathbf{P}_{\lambda} u_{l}=P\{A \otimes I, I \otimes B\}^{p-1}[P\{A \otimes I, I \otimes B\}-\lambda(I \otimes I)] u_{l} \rightarrow 0
$$

in $X \otimes Y$ as $l \rightarrow \infty$.

As $Y$ is of finite dimension, we can choose a sequence $\left\{y_{l}^{\prime}\right\}_{i=1}^{\infty}$ in $Y^{\prime}$ of unit vectors such that $\left\{\left\langle u_{l}, y_{l}^{\prime}\right\rangle_{Y}\right\}_{l=1}^{\infty}$ is not compact. Here set

$$
\left\langle u, y^{\prime}\right\rangle_{Y}=\sum_{i=1}^{r}\left\langle y_{i}, y^{\prime}\right\rangle x_{i},
$$

where $u=\sum_{i=1}^{r} x_{i} \otimes y_{i}$ in $X \otimes Y$ and $y^{\prime} \in Y^{\prime}$. We may assume, by taking subsequences, that $\left\{\left\langle u_{l}, y_{l}^{\prime}\right\rangle_{Y}\right\}_{l=1}^{\infty}$ is noncompact and bounded away from zero. Set

$$
x_{l}=\left\|\left\langle u_{l}, y_{l}^{\prime}\right\rangle_{Y}\right\|^{-1}\left\langle u_{l}, y_{l}^{\prime}\right\rangle_{Y}, \quad l=1,2, \ldots
$$

Then $\left\{x_{l}\right\}_{l=1}^{\infty}$ is a noncompact sequence of unit vectors in $D\left[A^{t m}\right]$.

To prove that $\lambda$ belongs to the right member of (3.9)' it suffices to show

$$
\left[P_{\lambda}(A, \nu)^{t} \otimes I\right] u_{l} \rightarrow 0, \quad P_{\lambda}(\xi, \nu)=P(\xi, \nu)-\lambda,
$$

in $X \otimes Y$ as $l \rightarrow \infty$. In fact, this implies $P_{\lambda}(A, \nu)^{t} x_{l} \rightarrow 0$ as $l \rightarrow \infty$, so that nul' $^{\prime} P_{\lambda}(A, v)^{t}=\infty$ and $0 \in \sigma_{e v}\left(P_{\lambda}(A, \nu)^{t}\right)$. By Proposition 1.2 there is a $\mu^{\prime} \in \sigma_{\text {ew }}(A)$ with $P\left(\mu^{\prime}, \nu\right)-\lambda=0$, whence follows the assertion.

Now we turn to the proof of $\left[P_{\lambda}(A, \nu)^{t} \otimes I\right] u_{l} \rightarrow 0$ as $l \rightarrow \infty$. The $t$-dimensional space $Y$ has the direct sum decomposition

$$
Y=N_{1} \oplus \cdots \oplus N_{t}
$$


such that

$$
N_{1} \oplus \cdots \oplus N_{k}=N\left[(B-\nu I)^{k}\right], \quad 1 \leqslant k \leqslant t
$$

Hence $X \otimes Y$ is decomposed into the topological direct sum

$$
X \otimes Y=\left(X \otimes N_{1}\right) \oplus \cdots \oplus\left(X \otimes N_{t}\right) \text {. }
$$

Then $u_{l}=\sum_{k=1}^{t} u_{k l}, u_{k l} \in X \otimes N_{k}, 1 \leqslant k \leqslant t, l=1,2, \ldots$ Each sequence $\left\{u_{k l}\right\}_{l=1}^{\infty}$ is bounded. We have to show for each $k, 1 \leqslant k \leqslant t$,

$$
\left[P_{\lambda}(A, \nu)^{l} \otimes I\right] u_{k l} \rightarrow 0 \text { as } l \rightarrow \infty \text {. }
$$

In fact, we show $\left[P_{\lambda}(A, \nu)^{p} \otimes I\right] u_{k l} \rightarrow 0$ as $l \rightarrow \infty$ for $t-(k-1)<p<t$. The proof is by induction on $k$ from $k=t$ to $k=1$.

For $k=t$, we see with the aid of (3.4) and Proposition 2.2(d) that for $1<p<t$,

$$
\begin{aligned}
& {\left[P_{\lambda}(A, \nu)^{p-1} \otimes(B-\nu I)^{t-1}\right][P\{A \otimes I, I \otimes B\}-\lambda(I \otimes I)] u_{l}} \\
& \quad=\left[P_{\lambda}(A, \nu)^{p} \otimes(B-\nu I)^{t-1}\right] u_{t l}
\end{aligned}
$$

approaches 0 in $X \otimes Y$ as $l \rightarrow \infty$. If $\left\{y_{i}\right\}_{i=1}^{i(t)}$ is a basis of $N_{t}$ then $u_{t l}=\sum_{i=1}^{i(t)} x_{i l}$ $\otimes y_{i}$, where $\left\{x_{i l}\right\}_{i=1}^{i(t)} \subset D\left[A^{t m}\right], l=1,2, \ldots$ We have only to consider the case $N_{t} \neq\{0\}$. Then $\left\{(B-\nu I)^{t-1} y_{i}\right\}_{i=1}^{i(t)}$ is linearly independent. It is easy to see that for $1 \leqslant i<i(t), P_{\lambda}(A, \nu)^{p} x_{i l}$ approaches 0 in $X$ as $l \rightarrow \infty$, whence $\left[P_{\lambda}(A, \nu)^{p} \otimes I\right] u_{t l} \rightarrow 0$ in $X \otimes Y$ as $l \rightarrow \infty$.

Assume now that the assertion is valid for $k>s, 1<s<t$. Similarly, for $t-(s-1)<p<t$,

$$
\begin{aligned}
{\left[P_{\lambda}(A, \nu)^{p-1} \otimes(B-\nu I)^{s-1}\right][P\{A \otimes I, I \otimes B\}-\lambda(I \otimes I)] u_{l} } \\
=\left[P_{\lambda}(A, \nu)^{p} \otimes(B-\nu I)^{s-1}\right] u_{s l} \\
+\left[P_{\lambda}(A, \nu)^{p-1} \otimes(B-\nu I)^{s-1}\right] \\
\\
\cdot[P\{A \otimes I, I \otimes B\}-\lambda(I \otimes I)] \sum_{h=s+1}^{t} u_{h l}
\end{aligned}
$$

approaches 0 in $X \otimes Y$ as $l \rightarrow \infty$.

First observe the second term above. By (3.4) rewrite it as

$$
\begin{gathered}
\left\{\left[\sum_{k=1}^{n}\left(\sum_{j=0}^{m} a_{j k} A^{j}\right) P_{\lambda}(A, \nu)^{p-1} \otimes(B-\nu I)^{s-1+k}\right]\right. \\
\left.+\left[P_{\lambda}(A, \nu)^{p} \otimes(B-\nu I)^{s-1}\right]\right\} \sum_{h=s+1}^{t} u_{h l}
\end{gathered}
$$


whose norm is, as easily seen, majorized by

$$
\sum_{h=s+1}^{t}\left(\left\|\left[P_{\lambda}(A, v)^{p} \otimes I\right] u_{h l}\right\|_{\alpha}+\left\|\left[P_{\lambda}(A, \nu)^{p-1} \otimes I\right] u_{h l}\right\|_{\alpha}\right) .
$$

Therefore by the induction hypothesis the second term concerned approaches 0 in $X \otimes Y$ as $l \rightarrow \infty$, since $t-(s-1) \leqslant p \leqslant t$. It follows from the first term that, for $t-(s-1) \leqslant p \leqslant t$,

$$
\left[P_{\lambda}(A, \nu)^{p} \otimes(B-\nu I)^{s-1}\right] u_{s l} \rightarrow 0
$$

as $l \rightarrow \infty$. The same argument as for $k=t$ yields that $\left[P_{\lambda}(A, \nu)^{p} \otimes I\right] u_{s l}$ approaches 0 in $X \otimes Y$ as $l \rightarrow \infty$ unless $N_{s} \neq\{0\}$. This proves the desired assertion.

In case def $^{\prime} \mathbf{P}_{\boldsymbol{\lambda}}$ is infinite, the same argument as above applies to

$$
P\left\{A^{\prime} \otimes I^{\prime}, I^{\prime} \otimes B^{\prime}\right\}-\lambda\left(I^{\prime} \otimes I^{\prime}\right)
$$

which is by Proposition 2.2(c) the adjoint of $\mathbf{P}_{\lambda}$, since the approximate deficiency of a densely defined closed linear operator coincides with the approximate nullity of its adjoint. There exists a noncompact sequence $\left\{u_{l}^{\prime}\right\}_{l=1}^{\infty}$ of unit vectors in $D\left[\left(A^{\prime}\right)^{l m}\right] \otimes Y^{\prime}$ such that for $1<p<t$

$$
P\left\{A^{\prime} \otimes I^{\prime}, I^{\prime} \otimes B^{\prime}\right\}^{p-1}\left[P\left\{A^{\prime} \otimes I^{\prime}, I^{\prime} \otimes B^{\prime}\right\}-\lambda\left(I^{\prime} \otimes I^{\prime}\right)\right] u_{l}^{\prime} \rightarrow 0
$$

in $X^{\prime} \otimes Y^{\prime}$ as $l \rightarrow \infty$. In the same way it can be shown that

$$
\left[P_{\lambda}\left(A^{\prime}, v\right)^{t} \otimes I^{\prime}\right] u_{l}^{\prime}
$$

approaches 0 in $X^{\prime} \otimes Y^{\prime}$ as $l \rightarrow \infty$. This implies that nul' $P_{\lambda}\left(A^{\prime}, \nu\right)^{t}=\infty$ and hence $\operatorname{def}^{\prime} P_{\lambda}(A, \lambda)^{t}=\infty$. Therefore by Proposition $1.2, \lambda$ belongs to the right member of (3.9)'.

II. The general case. Let $\lambda \in \sigma_{e w}(\mathrm{P})$. Since $P \in \mathscr{P}_{e}(A, B)$ there are nonempty open sets $U \supset \sigma(A), V \supset \sigma(B)$ and a large $r>0$ such that (2.4) holds. For this $r$ take the same open sets $U_{\varepsilon}$ and $V_{\varepsilon}$ as in the proof (b) of Theorem 3.1.

It suffices to show that for every sufficiently small $\varepsilon>0, \lambda$ belongs to the union of three sets

$$
P\left(\sigma_{e w}(A), \sigma(B) \backslash V_{\varepsilon}\right) \cup P\left(\sigma(A) \backslash U_{\varepsilon}, \sigma_{e w}(B)\right) \cup P\left(U_{\varepsilon}, V_{\varepsilon}\right) .
$$

In fact, suppose this is established. If, for some $\varepsilon>0, \lambda$ belongs to either of the first and second sets in (3.14) there is nothing more to prove. Therefore assume $\lambda$ belongs to $P\left(U_{\varepsilon}, V_{\varepsilon}\right)$ for every $\varepsilon>0$. For each positive integer $l$ choose $\left(\xi_{l}, \eta_{l}\right)$ in $\left(U_{1 / l} \cap K_{r}\right) \times\left(V_{1 / l} \cap K_{r}\right)$ with $P\left(\xi_{l}, \eta_{l}\right)=\lambda$. By the same reasoning as in the proof of Theorem 3.1 the sequence $\left\{\left(\xi_{l}, \eta_{l}\right)\right\}_{l=1}^{\infty}$ is bounded, so that we may assume it converges to $\left(\xi_{0}, \eta_{0}\right)$ as $l \rightarrow \infty$. Then $\left(\xi_{0}, \eta_{0}\right)$ lies in $\sigma_{e b}(A) \times \sigma_{e b}(B)$ and hence $\lambda=P\left(\xi_{0}, \eta_{0}\right)$ belongs to the set on 
the right of (3.10) and so on the right of (3.9), because they coincide as seen in the proof (a).

Now we come to the proof that $\lambda$ belongs to the set (3.14) for all $\varepsilon$. As in the proof of Theorem 3.1, let $\sigma(A) \backslash U_{e}=\left\{\alpha_{j}\right\}_{j=1}^{a}, \sigma(B) \backslash V_{\varepsilon}=\left\{\beta_{k}\right\}_{k=1}^{b}$, and let $P, Q$ be the projections associated with them. Then using the same notations as (2.5)-(2.8) we obtain

$$
\sigma_{e w}(\mathbf{P})=\sigma_{\text {ew }}\left(\mathbf{P}_{11}\right) \cup \sigma_{\text {ew }}\left(\mathbf{P}_{12}\right) \cup \sigma_{\text {ew }}\left(\mathbf{P}_{21}\right) \cup \sigma_{\text {ew }}\left(\mathbf{P}_{22}\right) \text {. }
$$

Since $P X$ and $Q Y$ are of finite dimension, $\sigma_{e w}\left(P_{11}\right)$ is empty. Since $P \in$ $\mathscr{P}_{e}(A, B)$, we have by Propositions 2.3 and 2.4 and by case $\mathrm{I}$,

$$
\begin{aligned}
& \sigma_{e w}\left(P_{12}\right)=P\left(\left\{\alpha_{j}\right\}_{j=1}^{a}, \sigma_{e w}\left(B_{2}\right)\right)=P\left(\sigma(A) \backslash U_{e}, \sigma_{e w}(B)\right), \\
& \sigma_{e w}\left(P_{21}\right)=P\left(\sigma_{e w}\left(A_{2}\right),\left\{\beta_{k}\right\}_{k=1}^{b}\right)=P\left(\sigma_{e w}(A), \sigma(B) \backslash V_{e}\right),
\end{aligned}
$$

and by Theorem 2.1,

$$
\sigma_{e w}\left(\mathrm{P}_{22}\right) \subset \sigma\left(\mathrm{P}_{22}\right)=P\left(\sigma\left(A_{2}\right), \sigma\left(B_{2}\right)\right) \subset P\left(U_{e}, V_{e}\right) .
$$

Thus putting all this together shows that $\sigma_{e w}(P)$ is included in (3.14). In particular, $\lambda$ belongs to (3.14). Q.E.D.

By Theorems 3.1 and 3.4, the intersection of the Browder essential spectrum and the Fredholm domain of $\tilde{P}\{A \otimes I, I \otimes B\}$ is determined.

COROLlaRY 3.5. The same hypothesis as in Theorem 3.4. Then

$$
\begin{aligned}
\sigma_{e b}(\tilde{P}\{A \otimes I, I \otimes B\}) \backslash \sigma_{e w}(\tilde{P}\{A \otimes I, I \otimes B\}) \\
=\left\{P\left(\sigma(A) \backslash \sigma_{e b}(A), \sigma_{e b}(B) \backslash \sigma_{e w}(B)\right)\right. \\
\left.\cup P\left(\sigma_{e b}(A) \backslash \sigma_{e w}(A), \sigma(B) \backslash \sigma_{e b}(B)\right)\right\} \\
\backslash\left\{P\left(\sigma_{e w}(A), \sigma(B)\right) \cup P\left(\sigma(A), \sigma_{e w}(B)\right)\right\} .
\end{aligned}
$$

For each $\lambda$ in the set (3.15) the sets (2.11), $\Delta_{10}(\lambda)$, and (2.12), $\Delta_{01}(\lambda)$, are finite and

$$
\begin{gathered}
\Delta_{10}(\lambda)=\Delta_{1}(\lambda) \cup \Delta_{12}(\lambda), \quad \Delta_{01}(\lambda)=\Delta_{1}(\lambda) \cup \Delta_{21}(\lambda), \\
\Delta_{0}(\lambda)=\Delta_{10}(\lambda) \cup \Delta_{01}(\lambda) .
\end{gathered}
$$

Proof. Obvious.

3.3. The nullity, deficiency and index. For $P \in \mathscr{P}_{e}(A, B)$, the operator (2.1), $\mathbf{P}_{\lambda}$, is Fredholm and at least one of nul $\mathbf{P}_{\lambda}$ and def $\mathbf{P}_{\lambda}$ does not vanish if and only if $\lambda$ is in the union of the sets (3.7) and (3.15) in Corollaries 3.3 and 3.5. This implies in view of (3.4) that if $\operatorname{dim} X=\infty$ then $P(\xi, \nu)-\lambda \nexists 0$ for each fixed $\nu \in \sigma(B) \backslash \sigma_{e b}(B)$, in which case we write

$$
P(\xi, \nu)-\lambda=d(\nu) \prod_{\mu ; P(\mu, \nu)=\lambda}(\xi-\mu)^{m(\mu, \nu)}, \quad d(\nu)=\sum_{k=0}^{n} c_{m(\nu), \nu^{\nu}},
$$


where $m(\nu)$ is the degree in $\xi$ of $P(\xi, \nu), m(\nu)=\Sigma_{\mu ; P(\mu, \nu)-\lambda} m(\mu, \nu) \leqslant m$. Similarly, if $\operatorname{dim} Y=\infty$ then $P(\mu, \eta)-\lambda \neq 0$ for each fixed $\mu \in \sigma(A) \backslash$ $\sigma_{e b}(A)$, in which case we write

$$
P(\mu, \eta)-\lambda=e(\mu) \prod_{\nu ; P(\mu, \nu)=\lambda}(\eta-\nu)^{n(\mu, \nu)}, \quad e(\mu)=\sum_{j=0}^{m} c_{j, n(\mu)} \mu^{j},
$$

where $n(\mu)$ is the degree in $\eta$ of $P(\mu, \eta), n(\mu)=\Sigma_{\nu ; P(\mu, \nu)-\lambda} n(\mu, \nu)<n$.

Set

$$
p(\mu, \nu)=t(B ; \nu) m(\mu, \nu), \quad q(\mu, \nu)=t(A ; \mu) n(\mu, \nu) .
$$

Let $r(p, q ; \mu, \nu)$ be the rank of the coefficient matrix of the system of the $p q$ linear equations with the $p q$ unknowns $u_{s t}$

$$
\sum_{\substack{1<j<s \\ 1<k<t}} b_{s-j, t-k} u_{j k}=0, \quad 1<s \leqslant p, 1<t<q,
$$

where the $b_{j k}$ are the coefficients of the Taylor expansion (3.3) of $P(\xi, \eta)$ at $(\mu, v)$. Set

$$
n(p, q ; \mu, \nu)=p q-r(p, q ; \mu, \nu) \geqslant 1 .
$$

We shall now establish explicit formulae for the nullity, deficiency and index of (2.1).

For a linear operator $T: D[T] \subset Z \rightarrow Z$ and a positive integer $p$ set

$$
\begin{aligned}
& n_{p}(T)=2 \operatorname{nul} T^{p}-\operatorname{nul} T^{p-1}-\operatorname{nul} T^{p+1}, \\
& d_{p}(T)=2 \operatorname{def} T^{p}-\operatorname{def} T^{p-1}-\operatorname{def} T^{p+1},
\end{aligned}
$$

when they are well defined. Here note (cf. [25, Lemma 3.3]) that nul $T^{p}$ (resp. def $T^{p}$ ) is finite if and only if nul $T$ (resp. def $T$ ) is finite.

The following result amplifies Corollaries 3.3 and 3.5.

THEOREM 3.6. Let $\alpha$ be a quasi-uniform reasonable norm on $X \otimes Y$ and let $P \in \mathscr{P}_{e}(A, B)$. Then for $\lambda$ in the set (3.7) or (3.15) the following formulae hold:

$$
\operatorname{nul}\left[\tilde{P}\{A \otimes I, I \otimes B\}-\lambda I \hat{\otimes}_{\alpha} I\right]
$$

$$
\begin{aligned}
&= \sum_{(\mu, \nu) \in \Delta_{1}(\lambda) \cup \Delta_{12}(\lambda) \cup \Delta_{21}(\lambda)} \sum_{p, q=1}^{\infty} n(p, q ; \mu, \nu) \tilde{n}_{p}(A-\mu I) \tilde{n}_{q}(B-\nu I) \\
& \operatorname{def}\left[\tilde{P}\{A \otimes I, I \otimes B\}-\lambda I \hat{\otimes}_{\alpha} I\right] \\
&=\sum_{(\mu, \nu) \in \Delta_{1}(\lambda) \cup \Delta_{12}(\lambda) \cup \Delta_{21}(\lambda)} \sum_{p, q=1}^{\infty} n(p, q ; \mu, \nu) \tilde{d}_{p}(A-\mu I) \tilde{d}_{q}(B-\nu I)
\end{aligned}
$$


$\operatorname{ind}\left[\tilde{P}\{A \otimes I, I \otimes B\}-\lambda I \hat{\otimes}_{\alpha} I\right]$

$$
\begin{aligned}
= & \sum_{(\mu, \nu) \in \Delta_{12}(\lambda)} \operatorname{ind}(B-\nu I) \sum_{p=1}^{t(A ; \mu)} n(p, q(\mu, \nu) ; \mu, \nu) n_{p}(A-\mu I) \\
& +\sum_{(\mu, \nu) \in \Delta_{21}(\lambda)} \operatorname{ind}(A-\mu I) \sum_{q=1}^{t(B ; \nu)} n(p(\mu, \nu), q ; \mu, \nu) n_{q}(B-\nu I) .
\end{aligned}
$$

Here for $(\mu, \nu) \in \Delta_{1}(\lambda)$,

$$
\begin{array}{cc}
\tilde{n}_{p}(A-\mu I)=n_{p}(A-\mu I), & \tilde{d}_{p}(A-\mu I)=d_{p}(A-\mu I), \\
& p=1,2, \ldots, \\
\tilde{n}_{q}(B-\nu I)=n_{q}(B-\nu I), & \tilde{d}_{q}(B-\nu I)=d_{q}(B-\nu I), \\
q=1,2, \ldots ;
\end{array}
$$

for $(\mu, \nu) \in \Delta_{12}(\lambda)$,

$$
\begin{aligned}
& \tilde{n}_{p}(A-\mu I)=n_{p}(A-\mu I), \quad \tilde{d}_{p}(A-\mu I)=d_{p}(A-\mu I), \\
& p=1,2, \ldots, \\
& \tilde{n}_{q}(B-\nu I)= \begin{cases}n_{q}(B-\nu I), & 1<q<q(\mu, \nu), \\
\operatorname{nul}(B-\nu I)^{q}-\operatorname{nul}(B-\nu I)^{q-1}, & q=q(\mu, \nu), \\
0, & q>q(\mu, \nu),\end{cases} \\
& \tilde{d}_{q}(B-\nu I)= \begin{cases}d_{q}(B-\nu I), & 1<q<q(\mu, \nu), \\
\operatorname{def}(B-\nu I)^{q}-\operatorname{def}(B-\nu I)^{q-1}, & q=q(\mu, \nu), \\
0, & q>q(\mu, \nu) ;\end{cases}
\end{aligned}
$$

and for $(\mu, \nu) \in \Delta_{21}(\lambda)$,

$$
\begin{aligned}
& \tilde{n}_{p}(A-\mu I)= \begin{cases}n_{p}(A-\mu I), & 1<p<p(\mu, \nu), \\
\operatorname{nul}(A-\mu I)^{p}-\operatorname{nul}(A-\mu I)^{p-1}, & p=p(\mu, v), \\
0, & p>p(\mu, \nu),\end{cases} \\
& \tilde{d}_{p}(A-\mu I)= \begin{cases}d_{p}(A-\mu I), & 1<p<p(\mu, \nu), \\
\operatorname{def}(A-\mu I)^{p}-\operatorname{def}(A-\mu I)^{p-1}, & p=p(\mu, \nu), \\
0, & p>p(\mu, \nu),\end{cases} \\
& \tilde{n}_{q}(B-\nu I)=n_{q}(B-\nu I), \tilde{d}_{q}(B-\nu I)=d_{q}(B-\nu I),
\end{aligned}
$$

Therefore the sum $\sum_{p, q=1}^{\infty}$ is finite and in fact taken over those $p$ and $q$ with 
$1<p<t(A ; \mu)$ and $1 \leqslant q \leqslant t(B ; \nu)$ for $(\mu, \nu) \in \Delta_{1}(\lambda)$, with $1<p<$ $t(A ; \mu)$ and $1 \leqslant q \leqslant q(\mu, \nu)$ for $(\mu, \nu) \in \Delta_{12}(\lambda)$, and with $1<p<p(\mu, \nu)$ and $1<q<t(B ; \nu)$ for $(\mu, \nu) \in \Delta_{21}(\lambda)$.

The proof of Theorem 3.6 will need a thorough investigation of the case in which either $X$ or $Y$ is of finite dimension. Let us keep in mind again Proposition 2.2.

First we consider the case where both $X$ and $Y$ are of finite dimension. In this case every polynomial $P(\xi, \eta)$ belongs to $\mathscr{P}_{e}(A, B)$.

Lemma 3.7. Let $\operatorname{dim} X<\infty$ and $\operatorname{dim} Y<\infty$. Then for every polynomial $P(\xi, \eta)$ and every $\lambda \in \mathrm{C}$

$$
\begin{aligned}
& \text { (3.22)' nul } \mathbf{P}_{\lambda}=\sum_{(\mu, \nu) \in \Delta_{0}(\lambda)} \sum_{p=1}^{t(A ; \mu)} \sum_{q=1}^{t(B ; \nu)} n(p, q ; \mu, \nu) n_{p}(A-\mu I) n_{q}(B-\nu I) \text {, } \\
& \text { (3.23) } \operatorname{def} \mathbf{P}_{\lambda}=\sum_{(\mu, \nu) \in \Delta_{0}(\lambda)} \sum_{p=1}^{t(A ; \mu)} \sum_{q=1}^{t(B ; \nu)} n(p, q ; \mu, \nu) d_{p}(A-\mu I) d_{q}(B-\nu I) \text {. }
\end{aligned}
$$

Proof. $A$ and $B$ may be considered as matrices. Both $\sigma(A)$ and $\sigma(B)$ consist of a finite number of eigenvalues. We show (3.22)'; (3.23)' follows from this, since the nullity and deficiency of a matrix coincide.

We may assume $A$ and $B$ are of the Jordan normal form. We have

$$
\begin{array}{ll}
X=\sum_{\mu \in \sigma(A)} \oplus X_{\mu}, & X_{\mu}=N\left[(A-\mu I)^{t(A ; \mu)}\right], \\
Y=\sum_{\nu \in \sigma(B)} \oplus Y_{\nu}, & Y_{\nu}=N\left[(B-\nu I)^{t(B ; \nu)}\right] .
\end{array}
$$

$A$ is decomposed by the $X_{\mu}$ and the part of $A$ in $X_{\mu}$ is the sum of $\mu I_{\mu}$ and a nilpotent matrix where $I_{\mu}$ is the identity matrix in $X_{\mu}$. The nullity of $(A-\mu I)^{s}$ for $1<s \leqslant t(A ; \mu)$ is equal to the nullity of its part in $X_{\mu}$. The same is true of $B$. It follows that

$$
X \otimes Y=\sum_{(\mu, \nu) \in \sigma(A) \times \sigma(B)} \oplus\left(X_{\mu} \otimes Y_{\nu}\right)
$$

and $\mathbf{P}$ is decomposed by the $X_{\mu} \otimes Y_{\nu}$. The part of $\mathbf{P}_{\lambda}$ in $X_{\mu} \otimes Y_{\nu}$ is one-to-one if $P(\mu, \nu) \neq \lambda$. The nullity of $\mathbf{P}_{\lambda}$ is the sum of the nullities of the parts of $P_{\lambda}$ in $X_{\mu} \otimes Y_{\nu}$ with $(\mu, \nu) \in \sigma(A) \times \sigma(B), P(\mu, \nu)=\lambda$.

Therefore, to establish (3.22)' it suffices to show that

$$
\operatorname{nulP}_{\lambda}=\sum_{s=1}^{p} \sum_{t=1}^{q} n(s, t ; \mu, \nu) n_{s}(A-\mu I) n_{t}(B-\nu I)
$$

in the case where 


$$
\begin{gathered}
X=N\left[(A-\mu I)^{p}\right], \quad \operatorname{dim} X=p=t(A ; \mu) \\
Y=N\left[(B-\nu I)^{q}\right], \quad \operatorname{dim} Y=q=t(B ; \nu) \\
\sigma(A)=\{\mu\}, \quad \sigma(B)=\{\nu\}, \quad P(\mu, \nu)=\lambda .
\end{gathered}
$$

In this case note that $A-\mu I$ and $B-\nu I$ are nilpotent.

First consider the special case in which $\operatorname{nul}(A-\mu I)=\operatorname{nul}(B-\nu I)=1$. In this case $A-\mu I$ (resp. $B-\nu I$ ) is a square matrix of order $p$ (resp. q) of the form

$$
\left[\begin{array}{llllll}
0 & & & & & \\
1 & 0 & & & & \\
& 1 & 0 & & & \\
0 & & & \cdot & \cdot & \\
0 & & & 1 & 0
\end{array}\right]
$$

Choose bases $\left\{e_{s}\right\}_{s=1}^{p}$ of $X$ and $\left\{f_{t}\right\}_{t=1}^{q}$ of $Y$ such that

$$
(A-\mu I) e_{s}=\left\{\begin{array}{ll}
e_{s+1}, & (s+1<p) \\
0, & (s=p),
\end{array} \quad(B-\nu I) f_{t}= \begin{cases}f_{t+1}, & (t+1<q) \\
0, & (t=q) .\end{cases}\right.
$$

Every $u$ in $X \otimes Y$ is represented as $u=\sum_{s=1}^{p} \sum_{t=1}^{q} u_{s t} e_{s} \otimes f_{t}$. We have

$$
\begin{aligned}
0 & =[P\{A \otimes I, I \otimes B\}-\lambda(I \otimes I)] u \\
& =\sum_{j+k>0} b_{j k}\left[(A-\mu I)^{j} \otimes(B-\nu I)^{k}\right] u \\
& =\sum_{\substack{1<s<p \\
1<t<q}} \sum_{\substack{1<j<s \\
1<k<t}} b_{s-j, t-k} u_{j k} e_{s} \otimes f_{t}
\end{aligned}
$$

in view of (3.3) and hence (3.19). Then with (3.20) we obtain

$$
\operatorname{nul} \mathbf{P}_{\lambda}=p q-r(p, q ; \mu, \nu) \equiv n(p, q ; \mu, \nu),
$$

which proves (3.28) because $n_{s}(A-\mu I)=\delta_{s p}, s=1,2, \ldots$, and $n_{t}(B-\nu I)$ $=\delta_{t q}, t=1,2, \ldots$.

In the general case in which $A-\mu I$ and $B-\nu I$ are nilpotent, $n_{s}(A-\mu I)$ is the number of the square matrices of order $s$ of the form (3.29) in the Jordan normal form of $A-\mu I$, and the same is true of $n_{t}(B-\nu I)$. This proves (3.28) in the general case and (3.22)'. Q.E.D.

In the following special cases, (a) is due to T. Ando.

COROLlary 3.8. The same hypothesis as in Lemma 3.7. 
(a)

$$
\begin{aligned}
& \operatorname{nul}(A \otimes I+I \otimes B-\lambda I \otimes I) \\
&=\sum_{\substack{(\mu, \nu) \in \sigma(A) \times \sigma(B) \\
\mu+\nu=\lambda}} \sum_{p=1}^{\infty}\left(\operatorname{nul}(A-\mu I)^{p}-\operatorname{nul}(A-\mu I)^{p-1}\right) \\
& \cdot\left(\operatorname{nul}(B-\nu I)^{p}-\operatorname{nul}(B-\nu I)^{p-1}\right) .
\end{aligned}
$$

(b) For $\lambda \neq 0$,

$$
\begin{aligned}
\operatorname{nul}(A \otimes B-\lambda I \otimes I) & \\
= & \sum_{\substack{(\mu, \nu) \in \sigma(A) \times \sigma(B) \\
\mu \nu=\lambda}} \sum_{p=1}^{\infty}\left(\operatorname{nul}(A-\mu I)^{p}-\operatorname{nul}(A-\mu I)^{p-1}\right) \\
& \cdot\left(\operatorname{nul}(B-\nu I)^{p}-\operatorname{nul}(B-\nu I)^{p-1}\right)
\end{aligned}
$$

and

$$
\operatorname{nul}(A \otimes B)=\operatorname{nul} A \cdot \operatorname{dim} Y+\operatorname{dim} X \cdot \operatorname{nul} B-\operatorname{nul} A \cdot \operatorname{nul} B .
$$

Here both the sums are finite.

Proof. We only note that in both the cases the $n(p, q ; \mu, \nu)$ in Lemma 3.7 are given by $n(p, q ; \mu, \nu)=\min (p, q)$. An elementary calculation will yield the formulae. Q.E.D.

Next we consider the case where one of $X$ and $Y$, say $Y$, is of finite dimension while the other, $X$, is of infinite dimension.

When $P(\xi, \nu)-\lambda \equiv 0$ for some $\nu \in \sigma(B)$, it is easy to see in view of (3.4) that both nul $\mathbf{P}_{\lambda}$ and def $\mathbf{P}_{\lambda}$ are infinite. Therefore the interest is in the case where $P(\xi, \nu)-\lambda \neq 0$ for each fixed $\nu \in \sigma(B)$. In this case $P(\xi, \nu)-\lambda$ is written as (3.16).

Lemma 3.9. Let $\operatorname{dim} X=\infty$ and $\operatorname{dim} Y<\infty$. Let $P \in \mathcal{P}_{e}(A, B)$. Assume that $P(\xi, \nu)-\lambda \neq 0$ for each fixed $\nu \in \sigma(B)$ or, in particular, that $\lambda$ does not belong to (3.9)'. Then:

(a)

(3.22)" nul $\mathbf{P}_{\lambda}=\sum_{(\mu, \nu) \in \Delta_{0}(\lambda)} \sum_{p=1}^{p(\mu, \nu)} \sum_{q=1}^{t(B ; \nu)} n(p, q ; \mu, \nu) \tilde{n}_{p}(A-\mu I) n_{q}(B-\nu I)$.

(b) Assume $R\left[\mathbf{P}_{\lambda}\right]$ is closed.

$$
(3.23)^{n} \quad \operatorname{def} \mathbf{P}_{\lambda}=\sum_{(\mu, \nu) \in \Delta_{0}(\lambda)} \sum_{p=1}^{p(\mu, \nu)} \sum_{q=1}^{t(B ; \nu)} n(p, q ; \mu, \nu) \tilde{d}_{p}(A-\mu I) d_{q}(B-\nu I) \text {. }
$$

Here the $\tilde{n}_{p}(A-\mu I)$ and $\tilde{d}_{p}(A-\mu I)$ are given by (3.27). 
Proof. (a) Recall (3.11). The proof is reduced to the proof of

$$
\left\{\begin{array}{l}
\sum_{\nu \in \sigma(B)} \oplus(N[P(A, \nu)-\lambda I] \otimes N[B-\nu I]) \subset N\left[\mathbf{P}_{\lambda}\right], \\
N\left[\mathbf{P}_{\lambda}\right] \subset \sum_{\nu \in \sigma(B)} \oplus\left(N\left[(P(A, \nu)-\lambda I)^{t(B ; \nu)}\right] \otimes Y_{\nu}\right) .
\end{array}\right.
$$

In fact, suppose this is established. Set $P_{\lambda}(\xi, \nu)=P(\xi, \nu)-\lambda$ again. It is readily seen from (3.30) in view of Propositions 1.2 and 1.4 that nul $P_{\lambda}$ is finite if and only if nul $P_{\lambda}(A, \nu)$ is finite for each $\nu \in \sigma(B)$ if and only if $\operatorname{nul}(A-\mu I)$ is finite for each $(\mu, \nu) \in \Delta_{0}(\lambda)$. In this case we show (3.30) yields (3.22)". Proposition 1.4 implies that for every positive integer $j$, $N\left[P_{\lambda}(A, \nu)^{t(B ; \nu)}\right]$ is a finite-dimensional subspace of $D\left[A^{j}\right]$ invariant under $A^{j}$. Consequently $N\left[P_{\lambda}(A, \nu)\right] \otimes Y_{\nu}$, which is a subspace of $D\left[A^{j}\right] \otimes Y$ for every positive integer $j$, is a finite-dimensional subspace of $X \otimes Y$ invariant under $\mathbf{P}_{\lambda}$. It follows that $\mathbf{P}_{\lambda}$ in $X \otimes Y$ and its restriction to the space on the right of the second inclusion in (3.30) have the same null space. Let $A_{\nu}$ be the part of $A$ in $N\left[P_{\lambda}(A, \nu)^{t(B ; \nu)}\right]$ and $B_{\nu}$ the part of $B$ in $Y_{\nu}$; the identity operators in both spaces are denoted by the same $I_{\nu}$. Then Lemma 3.7 with (3.30) yields

$$
\begin{aligned}
\operatorname{nul} \mathbf{P}_{\lambda} & =\sum_{\nu \in \sigma(B)} \operatorname{nul}\left(P\left\{A_{\nu} \otimes I_{\nu}, I_{\nu} \otimes B_{\nu}\right\}-\lambda\left(I_{\nu} \otimes I_{\nu}\right)\right) \\
& =\sum_{\nu \in \sigma(B)} \sum_{\substack{u \in \sigma\left(A_{\nu}\right) \\
P(\mu, \nu)=\lambda}} \sum_{p=1}^{p(\mu, \nu)} \sum_{q=1}^{t(B ; \nu)} n(p, q ; \mu, \nu) n_{p}\left(A_{\nu}-\mu I_{\nu}\right) n_{q}\left(B_{\nu}-\nu I_{\nu}\right) .
\end{aligned}
$$

For the upper bound $p(\mu, \nu)$ of the summation in $p$ in (3.31) note Remark at the end of $\$ 3.3$. Clearly $\sigma\left(A_{\nu}\right) \subset \sigma(A)$ and if $\mu$ is an eigenvalue of $A$ with $P(\mu, \nu)=\lambda$ then

$$
N[A-\mu I] \subset N\left[P_{\lambda}(A, \nu)\right] \subset N\left[P_{\lambda}(A, \nu)^{t(B ; \nu)}\right]
$$

by Proposition 1.4, whence $\mu \in \sigma\left(A_{\nu}\right)$. For every positive integer $q$, we have $n_{q}\left(B_{\nu}-\nu I_{\nu}\right)=n_{q}(B-\nu I)$ and

$$
n_{p}\left(A_{\nu}-\mu I_{\nu}\right)= \begin{cases}n_{p}(A-\mu I) & 1 \leqslant p<p(\mu, \nu), \\ \operatorname{nul}(A-\mu I)^{p}-\operatorname{nul}(A-\mu I)^{p-1}, & p=p(\mu, \nu), \\ 0, & p>p(\mu, \nu) .\end{cases}
$$

It follows that (3.31) is nothing but (3.22)".

Thus the proof of (3.22)" will be complete if (3.30) is established. In view of (3.4) the first inclusion in (3.30) is evident. Since $\mathbf{P}_{\boldsymbol{\lambda}}$ is decomposed by the $X \otimes Y_{\nu}$, to establish the second inclusion in (3.30) it suffices to show for each 
$\nu \in \sigma(B)$ that if $u$ is in $N\left[\mathbf{P}_{\lambda}\right] \cap\left(X \otimes Y_{\nu}\right)$ then $u$ is in $N\left[P_{\lambda}(A, \nu)^{t(B ; \nu)}\right] \otimes Y_{\nu}$.

To simplify the notation write $Y$ for $Y_{\nu}$ and $t$ for $t(B ; \nu)$. $Y$ has the direct sum decomposition (3.12) with (3.13). Then $u=\Sigma_{k=1}^{t} u_{k}$, where $u_{k}$ is in $D\left[A^{m}\right] \otimes N_{k}, 1 \leqslant k \leqslant t$. We show $u_{k}$ is in $N\left[P_{\lambda}(A, v)^{t-(k-1)}\right] \otimes N_{k}$.

The proof is by induction on $k$ from $k=t$ to $k=1$. For $k=t$ we have by (3.4)

$$
\begin{aligned}
0 & =\left[I \otimes(B-\nu I)^{t-1}\right][P\{A \otimes I, I \otimes B\}-\lambda(I \otimes I)] u \\
& =\left[P_{\lambda}(A, \nu) \otimes(B-\nu I)^{t-1}\right] u_{t} .
\end{aligned}
$$

If $N_{t} \neq\{0\}$, let $u_{t}=\sum_{i=1}^{i(t)} x_{i} \otimes y_{i}$, where the sequences $\left\{x_{i}\right\}_{i=1}^{i(t)} \subset D\left[A^{m}\right]$ and $\left\{y_{i}\right\}_{i=1}^{i(t)} \subset N_{t}$ are linearly independent. Since $(B-\nu I)^{t-1}$ is one-to-one on $N_{t}$ so that $\left\{(B-v I)^{t-1} y_{i}\right\}_{i=1}^{i(t)}$ is linearly independent, there exists a sequence $\left\{y_{i}^{\prime}\right\}_{i=1}^{i(t)} \subset Y^{\prime}$ with $\left\langle(B-\nu I)^{t-1} y_{i}, y_{j}^{\prime}\right\rangle=\delta_{i j}, i, j=1,2, \ldots, i(t)$. It follows that $P_{\lambda}(A, \nu) x_{i}=0$ for all $i$. Hence $u_{t}$ is in $N\left[P_{\lambda}(A, \nu)\right] \otimes N_{t}$. Assume the assertion is valid for $k>s, 1 \leqslant s<t$. Notice this implies in particular $\sum_{h=s+1}^{t} u_{h}$ belongs to $D\left[A^{j}\right] \otimes Y$ for every positive integer $j$. Then

$$
\begin{aligned}
0= & {\left[I \otimes(B-\nu I)^{s-1}\right][P\{A \otimes I, I \otimes B\}-\lambda(I \otimes I)] u } \\
= & {\left[P_{\lambda}(A, \nu) \otimes(B-\nu I)^{s-1}\right] u_{s} } \\
& +\left[I \otimes(B-\nu I)^{s-1}\right][P\{A \otimes I, I \otimes B\}-\lambda(I \otimes I)] \sum_{h=s+1}^{l} u_{h} .
\end{aligned}
$$

By the induction hypothesis the second term of the last equation above belongs to $N\left[P_{\lambda}(A, \nu)^{t-s}\right] \otimes Y$ and hence to $D\left[A^{j}\right] \otimes Y$ for every positive integer $j$. If $N_{s} \neq\{0\}$, let $u_{s}=\sum_{i=1}^{i(s)} x_{i} \otimes y_{i}$, where $\left\{x_{i}\right\}_{i=1}^{i(s)} \subset D\left[A^{m}\right]$ and $\left\{y_{i}\right\}_{i=1}^{i(s)} \subset N_{s}$ are linearly independent. Since $(B-\nu I)^{s-1}$ is one-to-one on $N_{s}$, the same argument as in the proof of Proposition 2.2(b) shows $\left\{P_{\lambda}(A, v) x_{i}\right\}_{i=1}^{i(s)}$ is included in $D\left[A^{j}\right]$ for every positive integer $j$. It follows that $\left\{x_{i}\right\}_{i=1}^{i(s)}$ is in $D\left[A^{j}\right]$ and hence $u_{s}$ is in $D\left[A^{j}\right] \otimes N_{s}$ for every positive integer $j$.

Thus we can apply $P_{\lambda}(A, \nu)^{t-s} \otimes I$ to the last equation above. Then we obtain by the induction hypothesis,

$$
\left[P_{\lambda}(A, \nu)^{t-(s-1)} \otimes(B-\nu I)^{s-1}\right] u_{s}=0 .
$$

Since $(B-\nu I)^{s-1}$ is one-to-one on $N_{s}$, the same argument as for $k=t$ above will show $u_{s}$ belongs to $N\left[P_{\lambda}(A, \nu)^{t-(s-1)}\right] \otimes N_{s}$. This proves (3.30).

(b) If $R\left[\mathbf{P}_{\lambda}\right]$ is closed then nul $\mathbf{P}_{\lambda}^{\prime}=\operatorname{def} \mathbf{P}_{\lambda}$. We note that $\sigma(A)=\sigma\left(A^{\prime}\right)$, $\sigma(B)=\sigma\left(B^{\prime}\right)$ and $P\left(A^{\prime}, v\right)=P(A, \nu)^{\prime}$. Although $D\left[A^{\prime}\right]$ is dense in $X^{\prime}$ in the weak* topology but not in the strong topology in general, the same argument as in (a) will justify that def $\mathbf{P}_{\lambda}$ (= nul $\mathbf{P}_{\lambda}^{\prime}$ ) is finite if and only if nul $P_{\lambda}\left(A^{\prime}, \lambda\right)$ is finite for each $\nu \in \sigma(B)$ if and only if $\operatorname{nul}\left(A^{\prime}-\mu I^{\prime}\right)$ is finite for each 
$(\mu, \nu) \in \Delta_{0}(\lambda)$. In this case, both $P_{\lambda}(A, \nu)$ and $A-\mu I$ necessarily have closed ranges, so that nul $P_{\lambda}\left(A^{\prime}, \nu\right)$ and $\operatorname{nul}\left(A^{\prime}-\mu I^{\prime}\right)$ can be replaced by $\operatorname{def} P_{\lambda}(A, \nu)$ and $\operatorname{def}(A-\mu I)$, respectively. In fact, otherwise both the approximate nullity and approximate deficiency of $P_{\lambda}(A, \nu)$ for some $\nu \in$ $\sigma(B)$ (resp. of $A-\mu I$ for some $(\mu, \nu) \in \Delta_{0}(\lambda)$ ) are infinite. Then since $\operatorname{nul}(B-\nu I)=\operatorname{def}(B-\nu I)>0$, by Lemma 3.2 and by closedness of $R\left[\mathbf{P}_{\lambda}\right]$ we obtain nul $\mathbf{P}_{\lambda}=$ def $\mathbf{P}_{\lambda}=\infty$, a contradiction.

The formula (3.23)" will be now obtained readily from (3.22)" with the above facts. Q.E.D.

Proof of THEOREM 3.6. I. The case where $\lambda$ is in the set (3.7). In this case note the sums for $(\mu, \nu)$ in $\Delta_{12}(\lambda)$ and $\Delta_{21}(\lambda)$ in (3.22) and (3.23) disappear. Following the proof of Corollary 3.3, we have nul $\mathbf{P}_{\lambda}=$ nul $\mathbf{P}_{\lambda 11}$ and def $\mathbf{P}_{\lambda}$ $=$ def $\mathbf{P}_{\lambda 11}$. Hence by Lemma 3.7 we have the formulae (3.22) and (3.23) with $\Delta_{12}(\lambda)$ and $\Delta_{21}(\lambda)$ deleted, since

$$
n_{p}\left(A_{1}-\mu I_{1}\right)=n_{p}(A-\mu I), \quad d_{p}\left(A_{1}-\mu I_{1}\right)=d_{p}(A-\mu I)
$$

and the same is true of $B$. It is clear that ind $\mathbf{P}_{\lambda}=0$.

II. The case where $\lambda$ is in the set (3.15). Denote the image of the projection of $\Delta_{10}(\lambda)$ (resp. $\Delta_{01}(\lambda)$ ) into the $\xi$ (resp. $\eta$ ) coordinate by $\Delta_{10}(\lambda ; A)$ (resp. $\Delta_{01}(\lambda ; B)$ ). Since $\mathbf{P}_{\lambda}$ is Fredholm, the parts $\mathbf{P}_{\lambda 11}, \mathbf{P}_{\lambda 12}, \mathbf{P}_{\lambda 21}$ and $\mathbf{P}_{\lambda 22}$ of $\mathbf{P}_{\lambda}$ relative to $\Delta_{10}(\lambda ; A)$ and $\Delta_{01}(\lambda ; B)$ are Fredholm. Since $P \in \mathscr{P}_{e}(A, B)$ it follows by Propositions 2.3 and 2.4 with Theorem 2.1 that

$$
\sigma\left(\mathrm{P}_{22}\right)=P\left(\sigma\left(A_{2}\right), \sigma\left(B_{2}\right)\right)=P\left(\sigma(A) \backslash \Delta_{10}(\lambda ; A), \sigma(B) \backslash \Delta_{01}(\lambda ; B)\right) .
$$

Consequently $\lambda \notin \sigma\left(\mathrm{P}_{22}\right)$ and

$$
\begin{aligned}
& \text { nul } \mathbf{P}_{\lambda}=\text { nul } \mathbf{P}_{\lambda 11}+\operatorname{nul} \mathbf{P}_{\lambda 12}+\operatorname{nul} \mathbf{P}_{\lambda 21}, \\
& \text { def } \mathbf{P}_{\lambda}=\operatorname{def} \mathbf{P}_{\lambda 11}+\operatorname{def} \mathbf{P}_{\lambda 12}+\operatorname{def} \mathbf{P}_{\lambda 21} .
\end{aligned}
$$

We apply Lemmas 3.7 and 3.9 to $\mathbf{P}_{\lambda 11}, \mathbf{P}_{\lambda 12}$ and $\mathbf{P}_{\lambda 21}$. Before this, note that

$$
\begin{aligned}
\Delta_{1}(\lambda) & =\left\{(\xi, \eta) \in \Delta_{10}(\lambda ; A) \times \Delta_{01}(\lambda ; B) ; P(\xi, \eta)=\lambda\right\}, \\
\Delta_{12}(\lambda) & =\left\{(\xi, \eta) \in \Delta_{10}(\lambda ; A) \times\left(\sigma(B) \backslash \Delta_{01}(\lambda ; B)\right) ; P(\xi, \eta)=\lambda\right\}, \\
\Delta_{21}(\lambda) & =\left\{(\xi, \eta) \in\left(\sigma(A) \backslash \Delta_{10}(\lambda ; A)\right) \times \Delta_{01}(\lambda ; B) ; P(\xi, \eta)=\lambda\right\} .
\end{aligned}
$$

Then we obtain

$$
\begin{aligned}
& \operatorname{nul} \mathbf{P}_{\lambda 11}=\sum_{(\mu, \nu) \in \Delta_{1}(\lambda)} \sum_{p=1}^{t(A ; \mu)} \sum_{q=1}^{t(B ; \nu)} n(p, q ; \mu, \nu) n_{p}\left(A_{1}-\mu I_{1}\right) n_{q}\left(B_{1}-\nu I_{1}\right), \\
& \operatorname{nul} \mathbf{P}_{\lambda 12}=\sum_{(\mu, \nu) \in \Delta_{12}(\lambda)} \sum_{p=1}^{t(A ; \mu)} \sum_{q=1}^{q(\mu, \nu)} n(p, q ; \mu, \nu) n_{p}\left(A_{1}-\mu I_{1}\right) \tilde{n}_{q}\left(B_{2}-\nu I_{2}\right), \\
& \operatorname{nul} \mathbf{P}_{\lambda 21}=\sum_{(\mu, \nu) \in \Delta_{21}(\lambda)} \sum_{p=1}^{p(\mu, \nu)} \sum_{q=1}^{t(B ; \nu)} n(p, q ; \mu, \nu) \tilde{n}_{p}\left(A_{2}-\mu I_{2}\right) n_{q}\left(B_{1}-\nu I_{1}\right) .
\end{aligned}
$$


However, for $\mu \in \sigma(\mathrm{A}) \backslash \sigma_{e b}(A), n_{p}\left(A_{1}-\mu I_{1}\right)=n_{p}(A-\mu I)$ and for $\mu \in$ $\sigma_{e b}(A) \backslash \sigma_{e w}(A), \tilde{n}_{p}\left(A_{2}-\mu I_{2}\right)=\tilde{n}_{p}(A-\mu I)$, and the same is true of $B$. This establishes (3.22).

Similarly, formula (3.23) for def $\mathbf{P}_{\boldsymbol{\lambda}}$ will be derived.

To get formula (3.24) for ind $\mathbf{P}_{\lambda}$ note that ind $\mathbf{P}_{\lambda}=$ ind $\mathbf{P}_{\lambda 12}+$ ind $\mathbf{P}_{\lambda 21}$, because nul $\mathbf{P}_{\lambda 11}=\operatorname{def} \mathbf{P}_{\lambda 11}$, and that

$$
\begin{aligned}
& \tilde{n}_{p}(A-\mu I)-\tilde{d}_{p}(A-\mu I)= \begin{cases}\operatorname{ind}(A-\mu I), & p=p(\mu, \nu), \\
0, & \text { otherwise; }\end{cases} \\
& \tilde{n}_{q}(B-\nu I)-\tilde{d}_{q}(B-\nu I)= \begin{cases}\operatorname{ind}(B-\nu I), & q=q(\mu, \nu), \\
0, & \text { otherwise. }\end{cases}
\end{aligned}
$$

Hence (3.24) follows. Q.E.D.

REMARK. The ascent $\alpha(T)$ (resp. descent $\delta(T)$ ) of $T$ is the smallest nonnegative integer $p$ such that $N\left[T^{p}\right]=N\left[T^{p+1}\right]$ (resp. $R\left[T^{p}\right]=R\left[T^{p+1}\right)$ ). If no such $p$ exists, set $\alpha(T)=\infty$ and $\delta(T)=\infty$ (see [24], [25]). Then $n_{p}(T)=0$ for $p>\alpha(T)$ and $d_{p}(T)=0$ for $p>\delta(T)$. If $\lambda$ is an isolated, finite-dimensional eigenvalue of $T$ then $\alpha(T-\lambda I)=\delta(T-\lambda I)<t(T ; \lambda)$.

A careful check will see that all $t(A ; \mu)$ and $t(B ; \nu)$ in Theorem 3.6, Lemmas 3.7 and 3.9 may be replaced by $\alpha(A-\mu I)$ and $\alpha(B-\mu I)$, respectively.

3.4. The Schechter essential spectrum.

THEOREM 3.10. Let $\alpha$ be a quasi-uniform reasonable norm on $X \otimes Y$ and let $P \in \mathscr{P}_{e}(A, B)$. Then $\sigma_{e m}(\tilde{P}\{A \otimes I, I \otimes B\})$ is the union of the set on the right of (3.9) and the set of all $\lambda$ contained in the set

$$
P\left(\sigma_{e m}(A), \sigma(B)\right) \cup P\left(\sigma(A), \sigma_{e m}(B)\right)
$$

but not in the set on the right of (3.9) such that the index (3.24) does not vanish.

Proof. In view of Theorem 3.4, Corollary 3.5 and Theorem 3.6, it suffices to show that every $\lambda$ in $\sigma_{e m}(P) \backslash \sigma_{e w}(P)$ belongs to (3.32). Since ind $P_{\lambda} \neq 0$, by (3.24) there is a pair $(\mu, \nu)$ in $\Delta_{12}(\lambda) \cup \Delta_{21}(\lambda)$ with $\operatorname{ind}(B-\nu I) \neq 0$ or $\operatorname{ind}(A-\mu I) \neq 0$. Hence either $\nu \in \sigma_{e m}(B)$ or $\mu \in \sigma_{e m}(A)$, in which case $\lambda=P(\mu, \nu)$ belongs to (3.32). Q.E.D.

3.5. Another polynomial operator. Assume $\alpha$ is in addition faithful on $X \otimes Y$. Associated with (0.1) is another polynomial operator $(0.3)$ in $X \hat{\otimes}_{\alpha} Y$. In this case, both (0.2) and (0.3) are also closable. Further, if $P \in \mathcal{P}_{e}(A, B)$, they have the same closure by [12, Theorem 3.1]; there it has been shown in fact for $P \in \mathscr{P}(A, B)$ but the same proof is valid in the present case. Therefore all the results in this section are valid for the closure of $(0.3)$. 
4. Two special cases. In this section the operators $(0.2)$ and $(0.3)$ in $X \hat{\otimes}_{\alpha} Y$ associated with the polynomials $P(\xi, \eta)=\xi+\eta$ and $P(\xi, \eta)=\xi \eta$ are considered. Throughout, $X$ and $Y$ are complex Banach spaces and $\alpha$ is a quasi-uniform reasonable norm on $X \otimes Y$.

We follow the same conventions as in $\S \S 2$ and 3.

We denote $\Delta_{0}(\lambda), \Delta_{1}(\lambda)$ and $\Delta_{j k}(\lambda)$ (in (2.9)-(2.14)) for $P(\xi, \eta)=\xi+\eta$ by $\Lambda_{0}(\lambda), \Lambda_{1}(\lambda)$ and $\Lambda_{j k}(\lambda)$, and for $P(\xi, \eta)=\xi \eta$ by $\Pi_{0}(\lambda), \Pi_{1}(\lambda)$ and $\Pi_{j k}(\lambda)$.

4.1. The case for $P(\xi, \eta)=\xi+\eta$. The corresponding operators are $A \otimes I$ $+I \otimes B$ and $A \hat{\otimes}_{\alpha} I+I \hat{\otimes}_{\alpha} B$, which we consider in the same situation as in $\$ 4$ of [12].

$Z$ being a complex Banach space, a densely defined closed linear operator $T: D[T] \subset Z \rightarrow Z$ is said to be of type $\left(\theta_{T}, M_{T}(\theta)\right), 0 \leqslant \theta_{T}<\pi$, if the resolvent set $\rho(T)$ includes the complementary set in $\mathrm{C}$ of the sector $S\left(\theta_{T}\right)=$ $\left\{\zeta ;|\arg \zeta| \leqslant \theta_{T}\right\}$ and $\left\|\zeta(\zeta I-T)^{-1}\right\| \leqslant M_{T}(\theta), \theta=\arg \zeta$, outside $S\left(\theta_{T}\right)$, where $M_{T}(\theta)$ is a constant depending only on $\theta=\arg \zeta$. In view of the resolvent equation, it is seen that if $T$ is of type $\left(\theta_{T}, M_{T}(\theta)\right)$ then for each fixed $\theta_{T}^{\prime}$ with $\theta_{T}<\theta_{T}^{\prime}<\pi,\left\|\zeta(\zeta I-T)^{-1}\right\|$ is uniformly bounded in the closure of $\operatorname{CS}\left(\theta_{T}^{\prime}\right)$.

We assume that $A$ and $B$ are respectively of type $\left(\theta_{A}, M_{A}(\theta)\right)$ and $\left(\theta_{B}, M_{B}(\theta)\right)$ with $0<\theta_{A}+\theta_{B}<\pi$ and that $A \otimes I+I \otimes B$ is closable in $X \hat{\otimes}_{\alpha} Y$ with closure $(A \otimes I+I \otimes B)^{2}$. In this case $\xi+\eta$ is in $\mathscr{P}_{e}(A, B)$.

THEOREM 4.1. (a) The Browder essential spectrum.

$$
\sigma_{e b}\left((A \otimes I+I . \otimes B)^{\sim}\right)=\left(\sigma_{e b}(A)+\sigma(B)\right) \cup\left(\sigma(A)+\sigma_{e b}(B)\right) .
$$

(b) The set of all isolated, finite-dimensional eigenvalues.

$$
\sigma\left((A \otimes I+I \otimes B)^{\sim}\right) \backslash \sigma_{e b}\left((A \otimes I+I \otimes B)^{\sim}\right)
$$

$$
\begin{aligned}
=\left\{\left(\sigma(A) \backslash \sigma_{e b}(A)\right)\right. & \left.+\left(\sigma(B) \backslash \sigma_{e b}(B)\right)\right\} \\
\backslash & \left\{\left(\sigma_{e b}(A)+\sigma(B)\right) \cup\left(\sigma(A)+\sigma_{e b}(B)\right)\right\} .
\end{aligned}
$$

If $\lambda$ is in the set (4.2) then

$$
t\left((A \otimes I+I \otimes B)^{\sim} ; \lambda\right)=\sum_{(\mu, \nu) \in \Lambda_{1}(\lambda)} t(A ; \mu) t(B ; \nu) .
$$

(c) The Wolf essential spectrum.

$$
\sigma_{e w}\left((A \otimes I+I \otimes B)^{\sim}\right)=\left(\sigma_{e w}(A)+\sigma(B)\right) \cup\left(\sigma(A)+\sigma_{e w}(B)\right) .
$$

(d) The intersection of the Browder essential spectrum and the Fredholm domain. 


$$
\begin{aligned}
\sigma_{e b}\left((A \otimes I+I \otimes B)^{\sigma}\right) \backslash \sigma_{e w}\left((A \otimes I+I \otimes B)^{\sim}\right) \\
=\left[\left\{\left(\sigma(A) \backslash \sigma_{e b}(A)\right)+\left(\sigma_{e b}(B) \backslash \sigma_{e w}(B)\right)\right\}\right. \\
\left.\cup\left\{\left(\sigma_{e b}(A) \backslash \sigma_{e w}(A)\right)+\left(\sigma(B) \backslash \sigma_{e b}(B)\right)\right\}\right] \\
\backslash\left\{\left(\sigma_{e w}(A)+\sigma(B)\right) \cup\left(\sigma(A)+\sigma_{e w}(B)\right)\right\} .
\end{aligned}
$$

(e) For $\lambda$ in the set (4.2) or (4.5) the following formulae hold:

$$
\begin{aligned}
& \operatorname{nul}\left((A \otimes I+I \otimes B)^{\sim}-\lambda I\right.\left.\hat{\otimes}_{\alpha} I\right) \\
&=\sum_{(\mu, \nu) \in \Lambda_{1}(\lambda) \cup \Lambda_{12}(\lambda) \cup \Lambda_{21}(\lambda)}\left\{\sum_{p=1}^{\infty}\left(\operatorname{nul}(A-\mu I)^{p}-\operatorname{nul}(A-\mu I)^{p-1}\right)\right. \\
&\left.\cdot\left(\operatorname{nul}(B-\nu I)^{p}-\operatorname{nul}(B-\nu I)^{p-1}\right)\right\} ;
\end{aligned}
$$

$\operatorname{def}\left((A \otimes I+I \otimes B)^{\sim}-\lambda I \hat{\otimes}_{\alpha} I\right)$

$$
\begin{aligned}
& \text { (4.7) }=\sum_{(\mu, \nu) \in \Lambda_{1}(\lambda) \cup \Lambda_{12}(\lambda) \cup \Lambda_{21}(\lambda)}\left\{\sum_{p=1}^{\infty}\left(\operatorname{def}(A-\mu I)^{p}-\operatorname{def}(A-\mu I)^{p-1}\right)\right. \\
& \left.\cdot\left(\operatorname{def}(B-\nu I)^{p}-\operatorname{def}(B-\nu I)^{p-1}\right)\right\} ;
\end{aligned}
$$

$\operatorname{ind}\left((A \otimes I+I \otimes B)^{-}-\lambda I \hat{\otimes}_{\alpha} I\right)$

$$
\begin{aligned}
(4.8)= & \sum_{(\mu, \nu) \in \Lambda_{12}(\lambda)} \operatorname{ind}(B-\nu I) \sum_{p=1}^{t(A ; \mu)}\left(\operatorname{nul}(A-\mu I)^{p}-\operatorname{nul}(A-\mu I)^{p-1}\right) \\
& +\sum_{(\mu, \nu) \in \Lambda_{21}(\lambda)} \operatorname{ind}(A-\mu I) \sum_{p=1}^{t(B ; \nu)}\left(\operatorname{nul}(B-\nu I)^{p}-\operatorname{nul}(B-\nu I)^{p-1}\right) .
\end{aligned}
$$

The sum $\sum_{p=1}^{\infty}$ is finite and in fact taken over those $p$ with $1<p<$ $\min (t(A ; \mu), t(B ; \nu))$ for $(\mu, \nu) \in \Lambda_{1}(\lambda)$, with $1<p<t(A ; \mu)$ for $(\mu, \nu) \in$ $\Lambda_{12}(\lambda)$ and with $1<p<t(B ; \nu)$ for $(\mu, \nu) \in \Lambda_{21}(\lambda)$. All $t(A ; \mu)$ and $t(B ; \nu)$ may be replaced by $\alpha(A-\mu I)$ and $\alpha(B-\nu I)$, respectively.

(f) The Schechter essential spectrum.

$$
\sigma_{e m}\left((A \otimes I+I \otimes B)^{\sim}\right)=\sigma_{1} \cup \sigma_{2},
$$

where $\sigma_{1}$ is the set on the right of (4.4) and $\sigma_{2}$ is the set of all $\lambda$ in the set

$$
\left\{\left(\sigma_{e m}(A)+\sigma(B)\right) \cup\left(\sigma(A)+\sigma_{e m}(B)\right)\right\} \backslash \sigma_{1}
$$

for which the index (4.8) does not vanish.

For $\alpha$ in addition faithful on $X \otimes Y$, so that $A \otimes I+I \otimes B$ and $A \hat{\otimes}_{\alpha} I$ 
$+I \hat{\otimes}_{\alpha} B$ are not only closable but also have the same closure in $X \hat{\otimes}_{\alpha} Y$, Theorem 4.1 is also valid with the closure $\left(A \hat{\otimes}_{\alpha} I+I \hat{\otimes}_{\alpha} B\right)^{-}$in place of $(A \otimes I+I \otimes B)^{\sim}$.

Theorem 4.1 enriches Theorem 4.6 in [12], where $X$ and $Y$ are Hilbert spaces, $\alpha$ is the prehilbertian norm $\sigma$, and $A$ and $B$ are $m$-sectorial operators with semiangles $\theta_{A}$ and $\theta_{B}$ with $0<\theta_{A}+\theta_{B}<\pi / 2$; in this case $A \hat{\otimes}_{0} I+I$ $\hat{\otimes}_{0} B$ is closed itself in $X \hat{\otimes}_{0} Y$.

4.2. The case for $P(\xi, \nu)=\xi \eta$. The corresponding operators are $A \otimes B$ and $A \hat{\otimes}_{\alpha} B$.

We assume that it is not the case that one of the extended spectra of $A$ and $B$ contains 0 while the other contains $\infty$, or, equivalently, that (i) $A$ and $B$ are bounded or (ii) $A$ and $B$ have everywhere defined bounded inverses or (iii) one of $A$ and $B$ is bounded and has an everywhere defined bounded inverse while the other is arbitrary (see [10], [11]). Further assume that $A \otimes B$ is closable in $X \hat{\otimes}_{\alpha} Y$ with closure $A \hat{\otimes}_{\alpha} B$ and that $\sigma(A) \cdot \sigma(B) \neq \mathbf{C}$. In this case $\xi \eta$ is in $\mathscr{P}_{e}(A, B)$.

THEOREM 4.2. (a) The Browder essential spectrum.

$$
\sigma_{e b}\left(A \hat{\otimes}_{\alpha} B\right)=\sigma_{e b}(A) \cdot \sigma(B) \cup \sigma(A) \cdot \sigma_{e b}(B) .
$$

(b) The set of all isolated, finite-dimensional eigenvalues.

$$
\begin{aligned}
\sigma\left(A \hat{\otimes}_{\alpha} B\right) \backslash \sigma_{e b}\left(A \hat{\otimes}_{\alpha} B\right) \\
=\left(\sigma(A) \backslash \sigma_{e b}(A)\right) \cdot\left(\sigma(B) \backslash \sigma_{e b}(B)\right) \\
\backslash\left(\sigma_{e b}(A) \cdot \sigma(B) \cup \sigma(A) \cdot \sigma_{e b}(B)\right) .
\end{aligned}
$$

If $\lambda \neq 0$ is in the set (4.11) then

$$
t\left(A \hat{\otimes}_{\alpha} B ; \lambda\right)=\sum_{(\mu, \nu) \in \Pi_{1}(\lambda)} t(A ; \mu) t(B ; \nu) .
$$

(c) The Wolf essential spectrum.

$$
\sigma_{e w}\left(A \hat{\otimes}_{\alpha} B\right)=\sigma_{e w}(A) \cdot \sigma(B) \cup \sigma(A) \cdot \sigma_{e w}(B) .
$$

(d) The intersection of the Browder essential spectrum and the Fredholm domain.

$$
\begin{aligned}
& \sigma_{e b}\left(A \hat{\otimes}_{\alpha} B\right) \backslash \sigma_{e w}\left(A \hat{\otimes}_{\alpha} B\right) \\
&=\left\{\left(\sigma(A) \backslash \sigma_{e b}(A)\right) \cdot\right.\left(\sigma_{e b}(B) \backslash \sigma_{e w}(B)\right) \\
&\left.\cup\left(\sigma_{e b}(A) \backslash \sigma_{e w}(A)\right) \cdot\left(\sigma(B) \backslash \sigma_{e b}(B)\right)\right\} \\
& \backslash\left(\sigma_{e w}(A) \cdot \sigma(B) \cup \sigma(A) \cdot \sigma_{e w}(B)\right) .
\end{aligned}
$$


(e) For $\lambda \neq 0$ in the set (4.11) or (4.14) the following formulae hold:

$$
\begin{aligned}
& \operatorname{nul}\left(A \hat{\otimes}_{\alpha} B-\lambda I \hat{\otimes}_{\alpha} I\right) \\
& \underset{(4.15)}{=} \sum_{(\mu, \nu) \in \Pi_{1}(\lambda) \cup \Pi_{12}(\lambda) \cup \Pi_{21}(\lambda)}\left\{\sum_{p=1}^{\infty}\left(\operatorname{nul}(A-\mu I)^{p}-\operatorname{nul}(A-\mu I)^{p-1}\right)\right. \\
& \left.\cdot\left(\operatorname{nul}(B-\nu I)^{p}-\operatorname{nul}(B-\nu I)^{p-1}\right)\right\} \\
& \operatorname{def}\left(A \hat{\otimes}_{\alpha} B-\lambda I \hat{\otimes}_{\alpha} I\right) \\
& \underset{(4.16)}{=} \sum_{(\mu, \nu) \in \Pi_{1}(\lambda) \cup \Pi_{12}(\lambda) \cup \Pi_{21}(\lambda)}\left\{\sum_{p=1}^{\infty}\left(\operatorname{def}(A-\mu I)^{p}-\operatorname{def}(A-\mu I)^{p-1}\right)\right. \\
& \left.\cdot\left(\operatorname{def}(B-\nu I)^{p}-\operatorname{def}(B-\nu I)^{p-1}\right)\right\}
\end{aligned}
$$

$\operatorname{ind}\left(A \hat{\otimes}_{\alpha} B-\lambda I \hat{\otimes}_{\alpha} I\right)$

$$
\begin{aligned}
= & \sum_{(\mu, \nu) \in \Pi_{12}(\lambda)} \operatorname{ind}(B-\nu I) \sum_{p=1}^{t(A ; \mu)}\left(\operatorname{nul}(A-\mu I)^{p}-\operatorname{nul}(A-\mu I)^{p-1}\right) \\
& +\sum_{(\mu, \nu) \in \Pi_{21}(\lambda)} \operatorname{ind}(A-\mu I) \sum_{D=1}^{t(B ; \nu)}\left(\operatorname{nul}(B-\nu I)^{p}-\operatorname{nul}(B-\nu I)^{p-1}\right) .
\end{aligned}
$$

The sum $\sum_{p=1}^{\infty}$ is finite and in fact taken over those $p$ with $1<p<$ $\min (t(A ; \mu), t(B ; \nu))$ for $(\mu, \nu) \in \Pi_{1}(\lambda)$, with $1<p<t(A ; \mu)$ for $(\mu, \nu) \in$ $\Pi_{12}(\lambda)$ and $1<p<t(B ; \nu)$ for $(\mu, \nu) \in \Pi_{21}(\lambda)$. All $t(A ; \mu)$ and $t(B ; \nu)$ may be replaced by $\alpha(A-\mu I)$ and $\alpha(B-\nu I)$, respectively.

(f) The Schechter essential spectrum.

$$
\sigma_{e m}\left(A \hat{\otimes}_{\alpha} B\right)=\sigma_{1} \cup \sigma_{2},
$$

where $\sigma_{1}$ is the set on the right of (4.13) and $\sigma_{2}$ is the set of all nonzero $\lambda$ in the set

$$
\left(\sigma_{e m}(A) \cdot \sigma(B) \cup \sigma(A) \cdot \sigma_{e m}(B)\right) \backslash \sigma_{1}
$$

for which the index (4.17) does not vanish.

REMARK 1. For the case $\lambda=0$ we make some comments. $\operatorname{nul}\left(A \hat{\otimes}_{\alpha} B\right)=0$ implies nul $A=\operatorname{nul} B=0$; the converse is valid if $\alpha$ is in addition faithful on $X \otimes Y$. In case nul $A>0$ or nul $B>0$, we have

$$
\operatorname{nul}\left(A \hat{\otimes}_{\alpha} B\right)=\operatorname{nul}(A \otimes B)=\operatorname{dim}(N[A] \otimes D[B]+D[A] \otimes N[B]),
$$

if either $X$ or $Y$ is of finite dimension, while otherwise $\operatorname{nul}\left(A \hat{\otimes}_{\alpha} B\right)=\infty$. An analogous argument is possible for $\operatorname{def}\left(A \hat{\otimes}_{\alpha} B\right)$ in case $R\left[A \hat{\otimes}_{\alpha} B\right]$ is closed. 
For $A \hat{\otimes}_{\pi} B$ closed, $R\left[A \hat{\otimes}_{\pi} B\right]$ is complementary if and only if both $R[A]$ and $R[B]$ are complementary, in which case $R\left[A \hat{\otimes}_{\pi} B\right]$ is the closure of $R[A] \otimes R[B]$ and $N\left[A \hat{\otimes}_{\pi} B\right]$ the closure of $N[A \otimes B]$ in $X \hat{\otimes}_{\pi} Y$. For both $X$ and $Y$ Hilbert spaces, $R\left[A \hat{\otimes}_{\sigma} B\right]$ is closed if and only if both $R[A]$ and $R[B]$ are closed, in which case $R\left[A \hat{\otimes}_{0} B\right]$ is the closure of $R[A] \otimes$ $R[B]$ and $N\left[A \hat{\otimes}_{\sigma} B\right]$ the closure of $N[A \otimes B]$ in $X \hat{\otimes}_{\sigma} Y$.

Remark 2. A very special case of $(4.15)$ is obtained by J. Piepenbrink and P. Rejto [19, (3.37)].

Acknowledgement. The author is indebted to Professor T. Ando for very useful discussions on the formula for the nullity as in Corollary 3.8(a). He also would like to thank Professor S. Koshi for several nice conversations.

Note. A part of the results was announced in the Seminars on "Tensor products of infinite-dimensional spaces" (September 26-28, 1974) and "Spectral and scattering theory and related topics" (January 30-February 1, 1975), both held at the Research Institute for Mathematical Sciences, Kyoto University [Brochure No. 228 (Japanese) and 242 (English)].

After the present paper had been accepted for publication it came to the attention of the author that M. Schechter and M. Snow used a different method to treat Theorems 3.1 and 3.4 for the special case where both $A$ and $B$ are bounded linear operators [M. Schechter and M. Snow, The Fredholm spectrum on tensor products, Proc. Roy. Irish Acad. Sect. A 75 (1975), 121-127; M. Snow, A joint Browder essential spectrum, Proc. Roy. Irish Acad. Sect. A 75 (1975), 129-131].

\section{REFERENCES}

1. E. Balslev and J. M. Combes, Spectral properties of many-body Schrödinger operators with dilatation-analytic interactions, Comm. Math. Phys. 22 (1971), 280-294. MR 49 \# 10288.

2. E. Balslev and T. W. Gamelin, The essential spectrum of a class of ordinary differential operators, Pacific J. Math. 14 (1964), 755-776. MR 30 \# 1410.

3. F. E. Browder, On the spectral theory of elliptic differential operators. I, Math. Ann. 142 (1960/61), 22-130. MR 35 \#804.

4. P. Enflo, A counterexample to the approximation problem in Banach spaces, Acta Math. 130 (1973), 309-317.

5. I. C. Gohberg and M. G. Krein, The basic propositions on defect numbers, root numbers and indices of linear operators, Uspehi Mat. Nauk 12 (1957), No. 2 (74), 43-118; English transl., Amer. Math. Soc. Transl. (2) 13 (1960), 185-264. MR 20 \#3459; 22 \#3984.

6. B. Gramsch and D. Lay, Spectral mapping theorems for essential spectra, Math. Ann. 192 (1971), 17-32. MR 45 \#936.

7. A. Grothendieck, Produits tensoriels topologiques et espaces nucléaires, Mem. Amer. Math. Soc. no. 16 (1955). MR 17, 763.

8. Résumé de la théorie métrique des produits tensoriels topologiques, Bol. Soc. Mat. São Paulo 8 (1956), 1-79. MR 20 \# 1194.

9. J. R. Holub, Compactness in topological tensor products and operator spaces, Proc. Amer. Math. Soc. 36 (1972), 398-406. MR 48 \#4802.

10. T. Ichinose, On the spectra of tensor products of linear operators in Banach spaces, J. Reine Angew. Math. 244 (1970), 119-153. MR 43 \#3828. 
11. Operational calculus for tensor products of linear operators in Banach spaces, Hokkaido Math. J. 4 (1975), no. 2, 306-334. MR 51 \#1154.

12. Operators on tensor products of Banach spaces, Trans. Amer. Math. Soc. 170 (1972), 197-219. MR 48 \#915.

13. M. A. Kaashoek and D. C. Lay, Ascent, descent, and commuting perturbations, Trans. Amer. Math. Soc. 169 (1972), 35-47. MR 47 \#861.

14. T. Kato, Perturbation theory for nullity, deficiency, and other quantities of linear operators, J. Analyse Math. 6 (1958), 261-322. MR 21 \#6541.

15. , Perturbation theory for linear operators, Springer-Verlag, Berlin and New York (1966). MR 34 \#3324.

16. D. Lay, Characterizations of the essential spectrum of F. E. Browder, Bull. Amer. Math. Soc. 74 (1968), 246-248. MR 36 \#4370.

17. D. J. Miličic and K. Veselić, On the boundary of essential spectra, Glasnik Mat. 6 (20) (1971), 73-78.

18. R. D. Nussbaum, Spectral mapping theorems and perturbation theorems for Browder's essential spectrum, Trans. Amer. Math. Soc. 150 (1970), 445-455. MR 42 \#876.

19. J. Piepenbrink and P. Rejto, Some singular Schrödinger operators with deficiency indices $\left(n^{2}\right.$, $n^{2}$ ), Duke Math. J. 41 (1974), 593-605. MR 50 \#752.

20. M. Reed and B. Simon, Tensor products of closed operators on Banach spaces, J. Functional Analysis 13 (1973), 107-124. MR 50 \# 1036.

21. R. Schatten, $A$ theory of cross-spaces, Ann. of Math. Studies, no. 26, Princeton Univ. Press, Princeton, N.J., 1950. MR 12, 186.

22. M. Schechter, On the essential spectrum of an arbitrary operator. I, J. Math. Anal. Appl. 13 (1966), 205-215. MR 32 \#6230.

23. B. Simon, Quadratic form techniques and the Balslev-Combes theorem, Comm. Math. Phys. 27 (1972), 1-9. MR 47 \#9989.

24. A. E. Taylor, Introduction to functional analysis, Wiley, New York, Chapman and Hall, London, 1958. MR 20 \#5411.

25. , Theorems on ascent, descent, nullity and defect of linear operators, Math. Ann. 163 (1966), 18-49. MR 32 \#8169.

26. F. Wolf, On the essential spectrum of partial differential boundary problems, Comm. Pure Appl. Math. 12 (1959), 211-228. MR 21 \#6472.

Department of Mathematics, HokKaido University, Sapporo, 060, Japan 